\title{
Synthesis of Vinyl-functionalized Oxazoles by Olefin Cross-Metathesis
}

\author{
Thomas J. Hoffman, ${ }^{a, b}$ James H. Rigby, ${ }^{b}$ Stellios Arseniyadis ${ }^{a}$ and Janine Cossy ${ }^{a *}$ \\ ${ }^{\mathrm{a}}$ Laboratoire de Chimie Organique, ESPCI, CNRS, 10 rue Vauquelin, 75231 Paris Cedex 05, France \\ ${ }^{\mathrm{b}}$ Department of Chemistry, Wayne State University, Detroit, Michigan 48202-3489
}

\section{Table of contents}

$\begin{array}{ll}\text { I. General Experimental } & \text { S2 }\end{array}$

II. Experimental and Spectral data of compounds 1, 2, 4a-n, 5a-m, and 9

S2-S13

III. Copies of ${ }^{1} \mathrm{H}$ NMR \& ${ }^{13} \mathrm{C}$ NMR

S14-S56 


\section{General Experimental:}

IR spectra were measured as thin films. ${ }^{1} \mathrm{H}$ NMR spectra were recorded at $400 \mathrm{MHz}$ in $\mathrm{CDCl}_{3}$ and data were reported as follows: chemical shift in parts per million from tetramethylsilane as an internal standard, multiplicity ( $\mathrm{s}=$ singlet, $\mathrm{d}=$ doublet, $\mathrm{t}=$ triplet, $\mathrm{q}=\mathrm{quartet}, \mathrm{m}=$ multiplet or overlap of nonequivalent resonances), integration. ${ }^{13} \mathrm{C} \mathrm{NMR}$ spectra were recorded at $100 \mathrm{MHz}$ in $\mathrm{CDCl}_{3}$ (unless otherwise specified) and data were reported as follows: chemical shift in parts per million from tetramethylsilane with the solvent as an internal indicator $\left(\mathrm{CDCl}_{3} \delta 77.0 \mathrm{ppm}\right)$, multiplicity with respect to proton (deduced from DEPT experiments, $\mathrm{s}=$ quaternary $\mathrm{C}, \mathrm{d}=\mathrm{CH}, \mathrm{t}=\mathrm{CH}_{2}, \mathrm{q}=\mathrm{CH}_{3}$ ). 1,4-Dioxane and THF was distilled from sodium/benzophenone. $\mathrm{CH}_{2} \mathrm{Cl}_{2}$ and toluene were distilled from $\mathrm{CaH}_{2}$. Other reagents were obtained from commercial suppliers and used as received. Analytical thin layer chromatography (TLC) was performed on silica gel plates visualized either with a UV lamp (254 nm), or by using solutions of $p$-anisaldehyde/sulfuric acid/acetic acid in EtOH or $\mathrm{KMnO}_{4} / \mathrm{K}_{2} \mathrm{CO}_{3}$ in water followed by heating. Flash chromatographies were performed on silica gel (230-400 mesh). Optical rotations were recorded on a digital polarimeter, $10 \mathrm{~mm}$ cell, and reported as $[\alpha]_{\mathrm{D}}^{20}(\mathrm{c}$ in $\mathrm{g}$ per $100 \mathrm{~mL}$ solvent). Mass spectra (MS) were obrained using an ESI mass spectrometer. All the reactions were carried out under $\mathrm{N}_{2}$ atmosphere. Organic extracts were dried over anhydrous $\mathrm{MgSO}_{4}$.

\section{Experimental and Spectral data of compounds 1, 2, 4a-n, 5a-m, and 9}

\section{2-Vinyloxazole-4-carboxylic acid ethyl ester 1.}

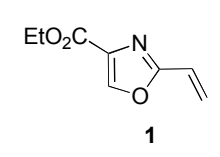

To a stirred suspension of acrylamide $\left(2.41 \mathrm{~g}, 33.9 \mathrm{mmol}\right.$ ) and finely ground $\mathrm{NaHCO}_{3}$ (oven-dried overnight at $110{ }^{\circ} \mathrm{C}$ ) in $\mathrm{THF}$ was added ethyl bromopyruvate (5.2 mL, $37.0 \mathrm{mmol}, 1.0$ equiv). The 
reaction mixture was then allowed to gradually reach $57^{\circ} \mathrm{C}$ and was heated overnight. After $12 \mathrm{~h}$, an additional amount of ethyl bromopyruvate $(5.2 \mathrm{~mL}, 37.0 \mathrm{mmol}, 1.0$ equiv) was added and heating was continued for $8 \mathrm{~h}$. The reaction mixture was allowed to reach $\mathrm{rt}$, the solid impurities were removed through filtration over Celite and the solvent was concentrated under reduced pressure. The crude residue was dissolved in THF $(15 \mathrm{~mL})$ and cooled to $0{ }^{\circ} \mathrm{C}$ before trifluoroacetic anhydride $(15 \mathrm{~mL}$, 101.9 mmol, 3.2 equiv) was added dropwise. The resultant solution was allowed to reach rt overnight. The reaction mixture was then slowly quenched with a saturated $\mathrm{NaHCO}_{3}$ solution $(30 \mathrm{~mL})$ and the organic layer was extracted with EtOAc $(3 \times 75 \mathrm{~mL})$. The combined organic layers were washed with brine, dried over MgSO4, and concentrated under reduced pressure. The crude product was puified by column chromatography on silica gel using a gradient of eluent $(9 / 1-7 / 3$ : EtOAc/hexanes) to provide ethyl 2-vinyloxazole-4-carboxylate (1) as a yellowish oil (3.06 g, 72\%); IR: $v=3156,2984,1739,1721$, 1312, 1144, 1116, 757; ${ }^{1} \mathrm{H}$ NMR (400 MHz, $\left.\mathrm{CDCl}_{3}\right) \delta 8.16(\mathrm{~s}, 1 \mathrm{H}), 6.64(\mathrm{dd}, J=17.8,11.3 \mathrm{~Hz}, 1 \mathrm{H})$, $6.28(\mathrm{dd}, J=17.6,0.5 \mathrm{~Hz}, 1 \mathrm{H}), 5.74(\mathrm{dd}, J=11.3,0.8,1 \mathrm{H}), 4.38(\mathrm{q}, J=7.0 \mathrm{~Hz}, 2 \mathrm{H}), 1.37(\mathrm{t}, J=7.3$ $\mathrm{Hz}) ;{ }^{13} \mathrm{C}$ NMR (100 MHz, $\left.\mathrm{CDCl}_{3}\right) \delta 161.8$ (s), 161.4 (s), 143.7 (d), 134.5 (s), 124.7 (d), 122.8 (t), 61.7

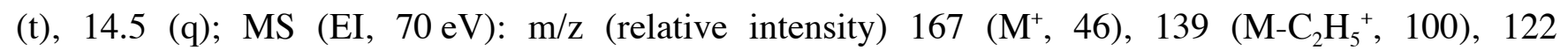
$\left(\mathrm{M}-\mathrm{C}_{2} \mathrm{H}_{6} \mathrm{O}^{+}, 40\right), 111$ (17), $94\left(\mathrm{M}_{-} \mathrm{C}_{4} \mathrm{H}_{5} \mathrm{O}_{2}^{+}, 66\right.$ (11), 55 (74); HRMS (ESI) calcd for $\mathrm{C}_{8} \mathrm{H}_{9} \mathrm{O}_{3} \mathrm{~N}$ $[\mathrm{M}+\mathrm{Na}]^{+}: 190.0475$, found: 190.0469.

General Procedure for the CM of vinyl-functionalized oxazoles with various olefins. To a stirred solution of 2 - or 4-vinyl-functionalized oxazole $(0.11 \mathrm{mmol})$ and the selected olefin $(0.17 \mathrm{mmol}$, 1.5 equiv) in $\mathrm{CH}_{2} \mathrm{Cl}_{2}(1 \mathrm{~mL})$ was added either [Ru]-II or [Ru]-III $(0.01 \mathrm{mmol}, 10 \mathrm{~mol} \%)$. The reaction mixture was heated under an argon atmosphere at $40{ }^{\circ} \mathrm{C}$ until completion (the reactions were monitored by TLC). The solvent was then removed under reduced pressure and the crude residue was purified by flash column chromatography on silica gel using a gradient of eluent $\left(\mathrm{Et}_{2} \mathrm{O} / n\right.$-pentane: $1 / 9$ to $\left.5 / 5\right)$ or (EtOAc/hexanes: 5/95 to 5/5) to provide the corresponding products (see Tables 1-2). 
(E)-2-(4-Methylpent-1-enyl)oxazole-4-carboxylic acid ethyl ester 4a.

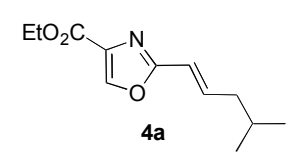

IR: $v=2958,2871,1744,1721,1315,1145,1112 ;{ }^{1} \mathrm{H} \mathrm{NMR}\left(400 \mathrm{MHz}, \mathrm{CDCl}_{3}\right) \delta 8.10(\mathrm{~s}, 1 \mathrm{H}), 6.80(\mathrm{dt}$, $J=16.1,7.5 \mathrm{~Hz}, 1 \mathrm{H}), 6.28(\mathrm{dt}, J=16.1,1.3 \mathrm{~Hz}, 1 \mathrm{H}), 4.37(\mathrm{q}, J=7.3 \mathrm{~Hz}, 2 \mathrm{H}), 2.15(\mathrm{dd}, J=10.9,1.3$ $\mathrm{Hz}, 1 \mathrm{H}), 2.13(\mathrm{dd}, J=10.9,1.3 \mathrm{~Hz}, 1 \mathrm{H}), 1.76$ (septuplet, $J=6.8 \mathrm{~Hz}, 1 \mathrm{H}), 1.37(\mathrm{t}, J=7.0,3 \mathrm{H}), 0.93(\mathrm{~d}$, $J=6.8 \mathrm{~Hz}, 6 \mathrm{H}) ;{ }^{13} \mathrm{C}$ NMR $\left(100 \mathrm{MHz}, \mathrm{CDCl}_{3}\right) \delta 162.1$ (s), 161.7 (s), $143.2(\mathrm{~d}), 141.8$ (d), 134.5 (d), 117.1 (d), 61.5 (t), 42.4 (t), 28.4 (q), 22.6 (d), 14.6 (q); MS (EI, 70 eV): m/z (relative intensity) $223\left(\mathrm{M}^{+}\right.$,

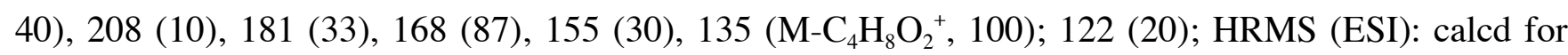
$\mathrm{C}_{12} \mathrm{H}_{17} \mathrm{O}_{3} \mathrm{~N}[\mathrm{M}+\mathrm{Na}]^{+}:$246.1101, found: 246.1094.

(E)-2-(3-(Trimethylsilanyl)prop-1-enyl)oxazole-4-carboxylic acid ethyl ester 4 b.

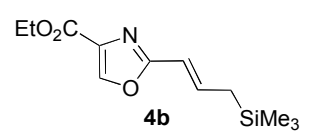

$\mathrm{mp}=51-53{ }^{\circ} \mathrm{C} ; \mathrm{IR}: v=2956,1744,1720,1315,1146,1112,856 ;{ }^{1} \mathrm{H} \mathrm{NMR}\left(400 \mathrm{MHz}, \mathrm{CDCl}_{3}\right) \delta 8.08$ $(\mathrm{s}, 1 \mathrm{H}), 6.87(\mathrm{dt}, J=15.8,8.8 \mathrm{~Hz}, 1 \mathrm{H}), 6.15(\mathrm{dt}, J=16.1 \mathrm{~Hz}, 1 \mathrm{H}), 4.38(\mathrm{q}, J=7.3 \mathrm{~Hz}, 2 \mathrm{H}), 1.76(\mathrm{dd}$, $J=8.8,1.3 \mathrm{~Hz}, 2 \mathrm{H}), 1.37(\mathrm{t}, J=7.3 \mathrm{~Hz}, 3 \mathrm{H}), 0.06(\mathrm{~s}, 9 \mathrm{H}) ;{ }^{13} \mathrm{C} \mathrm{NMR}\left(100 \mathrm{MHz}, \mathrm{CDCl}_{3}\right) \delta 162.6(\mathrm{~s})$, $161.9(\mathrm{~s}), 142.9,(\mathrm{~d}), 141.0(\mathrm{~d}), 134.3(\mathrm{~s}), 114.1(\mathrm{~s}), 61.5(\mathrm{t}), 25.5(\mathrm{t}), 14.6(\mathrm{q}),-1.53(\mathrm{q}) ; \mathrm{MS}(\mathrm{EI}, 70 \mathrm{eV})$ : m/z (relative intensity) $253\left(\mathrm{M}^{+}, 24\right), 238\left({\left.\mathrm{M}-\mathrm{CH}_{3}{ }^{+}, 13\right)}^{2} 25\left(\mathrm{M}-\mathrm{C}_{2} \mathrm{H}_{2}^{+}, 25\right), 208\left(\mathrm{M}-\mathrm{C}_{2} \mathrm{H} 5 \mathrm{O}+, 9\right), 135\right.$ $\left(\mathrm{M}-\mathrm{C}_{5} \mathrm{H}_{14} \mathrm{SiO}^{+}, 100\right), 107$ (18); HRMS (ESI): calcd for $\mathrm{C}_{12} \mathrm{H}_{19} \mathrm{O}_{3} \mathrm{NSi}[\mathrm{M}+\mathrm{Na}]^{+}:$276.1026, found: 276.1021.

(E)-2-(4-Bromobut-1-enyl)oxazole-4-carboxylic acid ethyl ester 4c.

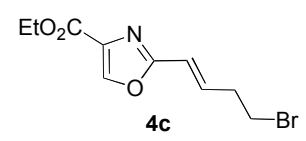


$\mathrm{mp}=46-48{ }^{\circ} \mathrm{C} \mathrm{IR}: v=3158,2982,2917,1738,1720,1315,1113 ;{ }^{1} \mathrm{H} \mathrm{NMR}\left(400 \mathrm{MHz}, \mathrm{CDCl}_{3}\right) \delta 8.14$ $(\mathrm{s}, 1 \mathrm{H}), 6.78(\mathrm{dt}, J=16.3,4.5 \mathrm{~Hz}, 1 \mathrm{H}), 6.42(\mathrm{dt}, J=16.1,1.5 \mathrm{~Hz}, 1 \mathrm{H}), 4.38(\mathrm{q}, J=7.0 \mathrm{~Hz}, 2 \mathrm{H}), 3.48(\mathrm{t}$, $J=6.8 \mathrm{~Hz}, 2 \mathrm{H}), 2.85(\mathrm{dd}, J=6.8,1.5 \mathrm{~Hz}, 1 \mathrm{H}), 2.82(\mathrm{dd}, J=6.8,1.5 \mathrm{~Hz}, 1 \mathrm{H}), 1.38(\mathrm{t}, J=7.3 \mathrm{~Hz}, 3 \mathrm{H})$; ${ }^{13} \mathrm{C}$ NMR (100 MHz, $\left.\mathrm{CDCl}_{3}\right) \delta 161.6(\mathrm{~s}), 161.4(\mathrm{~s}), 143.6$ (d), 138.2 (d), 134.7 (s), 118.6 (d), 61.6 (t), $36.0(\mathrm{t}), 30.8$ (t), 14.6 (q); MS (EI, $70 \mathrm{eV}): \mathrm{m} / \mathrm{z}$ (relative intensity) $275\left(\left(\mathrm{Br}^{81}\right) \mathrm{M}^{+}, 50\right) 273\left(\left(\mathrm{Br}^{79}\right) \mathrm{M}^{+}\right.$ 51), $245\left(\left(\mathrm{Br}^{81}\right) \mathrm{M}-\mathrm{C}_{2} \mathrm{H}_{4}^{+}, 56\right), 243\left(\left(\mathrm{Br}^{79}\right) \mathrm{M}-\mathrm{C}_{2} \mathrm{H}_{4}^{+}, 57\right), 230\left(\left(\mathrm{Br}^{81}\right) \mathrm{M}-\mathrm{C}_{2} \mathrm{H}_{4} \mathrm{O}^{+}, 29\right) 229\left(\left(\mathrm{Br}^{79}\right) \mathrm{M}-\mathrm{C}_{2} \mathrm{H}_{4} \mathrm{O}^{+}\right.$,

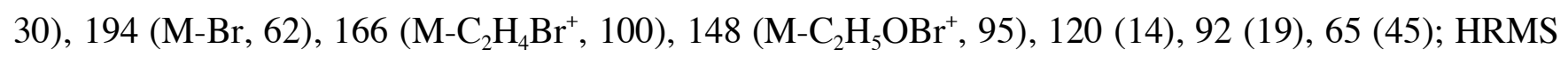
(ESI): calcd for $\mathrm{C}_{10} \mathrm{H}_{12} \mathrm{O}_{3} \mathrm{NBr}[\mathrm{M}+\mathrm{Na}]^{+}:\left(\mathrm{Br}^{81}\right)$ 297.9873, $\left(\mathrm{Br}^{79}\right)$ 295.9893, found: $\left(\mathrm{Br}^{81}\right)$ 297.9865, $\left(\mathrm{Br}^{79}\right) 295.9889$.

(E)-2-[3-(4-Ethoxycarbonyloxazol-2-yl)allyl]malonic acid dimethyl ester 4d.

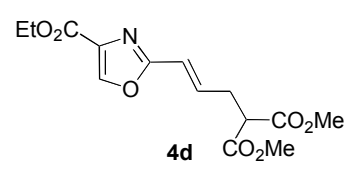

IR: $v=2956,1736,1315,1233,1152,1134 ;{ }^{1} \mathrm{H}$ NMR $\left(400 \mathrm{MHz}, \mathrm{CDCl}_{3}\right) \delta 8.11(\mathrm{~s}, 1 \mathrm{H}), 6.76(\mathrm{dt}$, $J=16.3,7.3 \mathrm{~Hz}, 1 \mathrm{H}), 6.40(\mathrm{dt}, J=16.0,1.5 \mathrm{~Hz}, 1 \mathrm{H}), 4.38(\mathrm{q}, J=7.3 \mathrm{~Hz}, 2 \mathrm{H}), 3.75(\mathrm{~s}, 1 \mathrm{H}), 3.55(\mathrm{t}$, $J=7.3 \mathrm{~Hz}, 1 \mathrm{H}), 2.85(\mathrm{td}, J=7.3,1.5 \mathrm{~Hz}, 2 \mathrm{H}), 1.37(\mathrm{t}, J=7.0 \mathrm{~Hz}, 3 \mathrm{H}) ;{ }^{13} \mathrm{C} \mathrm{NMR}\left(100 \mathrm{MHz}, \mathrm{CDCl}_{3}\right)$ $\delta 169.0(\mathrm{~s}), 161.6(\mathrm{~s}), 161.3(\mathrm{~s}), 143.5(\mathrm{~d}), 137.1(\mathrm{~d}), 134.6(\mathrm{~s}), 118.8(\mathrm{~d}), 61.6(\mathrm{t}), 53.1(\mathrm{q}), 51.0(\mathrm{~d})$, $32.1(\mathrm{t}), 14.6$ (q); MS (EI, $70 \mathrm{eV}): \mathrm{m} / \mathrm{z}$ (relative intensity) 331, $\left(\mathrm{M}^{+}, 1\right), 282\left(\mathrm{M}-\mathrm{CH}_{3} \mathrm{OH}^{+}, 35\right), 247$ (M- $\left.\mathrm{C}_{2} \mathrm{H}_{8} \mathrm{O}_{2}^{+}, 100\right), 220$ (27), 206 (45), 164 (30); HRMS (ESI): calcd for $\mathrm{C}_{14} \mathrm{H}_{17} \mathrm{O}_{7} \mathrm{~N}[\mathrm{M}+\mathrm{Na}]^{+}$: 334.0897, found: 334.0889 .

(E)-2-(4-(4-Methoxybenzyloxy)but-1-enyl)oxazole-4-carboxylic acid ethyl ester 4e.

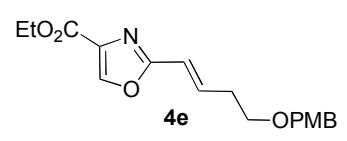


IR: $v=2855,1740,1719,1513,1314,1245,1111 \mathrm{~cm}^{-1} ;{ }^{1} \mathrm{H}$ NMR $\left(400 \mathrm{MHz}, \mathrm{CDCl}_{3}\right) \delta 8.11(\mathrm{~s}, 1 \mathrm{H})$, 7.27-7.23 (m, 2H), 6.90-6.84 (m, 2H), $6.83\left(\mathrm{t}_{\text {app }}, J=7.0 \mathrm{~Hz}, 1 \mathrm{H}\right), 6.38(\mathrm{dt}, J=16.0 \mathrm{~Hz}, 1.8 \mathrm{~Hz}, 1 \mathrm{H})$, $4.45(\mathrm{~s}, 2 \mathrm{H}), 4.38(\mathrm{q}, J=7.3 \mathrm{~Hz}, 2 \mathrm{H}), 3.79(\mathrm{~s}, 3 \mathrm{H}), 3.57\left(\mathrm{t}_{\mathrm{app}}, J=6.5 \mathrm{~Hz}, 2 \mathrm{H}\right), 2.57(\mathrm{dd}, J=6.5,1.5 \mathrm{~Hz}$ 1H), $2.53(\mathrm{dd}, J=6.5,1.5 \mathrm{~Hz}, 1 \mathrm{H}), 1.38(\mathrm{t}, J=7 \mathrm{~Hz}, 3 \mathrm{H}) ;{ }^{13} \mathrm{C} \mathrm{NMR}\left(100 \mathrm{MHz}, \mathrm{CDCl}_{3}\right) \delta 161.9(\mathrm{~s})$, 161.7 (s), 159.5 (s), 143.3 (d), 139.3 (d), 134.5 (d), 130.5 (s), 129.6 (s), 117.6 (d), 114.1 (d), 73.1 (t), $68.6(\mathrm{t}), 61.5(\mathrm{t}), 55.6(\mathrm{q}), 33.5(\mathrm{t}), 14.6(\mathrm{q}) ; \mathrm{MS}(\mathrm{EI}, 70 \mathrm{eV}): \mathrm{m} / \mathrm{z}$ (relative intensity) $331\left(\mathrm{M}^{+}, 2\right), 286$

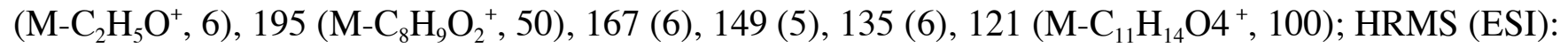
calcd for $\mathrm{C}_{18} \mathrm{H}_{21} \mathrm{O}_{5} \mathrm{~N}[\mathrm{M}+\mathrm{Na}]^{+}: 354.1312$, found: 354.1306 .

(士)-(E)-2-(4-Oxiranyl)but-1-enyl)oxazole-4-carboxylic acid ethyl ester 4 f.

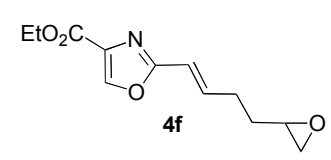

IR: $v=2923,1740,1719,1316,1423,1112 ;{ }^{1} \mathrm{H}$ NMR $\left(400 \mathrm{MHz}, \mathrm{CDCl}_{3}\right) \delta 8.12(\mathrm{~s}, 1 \mathrm{H}), 6.85(\mathrm{dt}$, $J=16.1,7.0 \mathrm{~Hz}, 1 \mathrm{H}), 6.36(\mathrm{dt}, J=16.0,1.0 \mathrm{~Hz}, 1 \mathrm{H}), 4.40(\mathrm{q}, J=7.0 \mathrm{~Hz}, 2 \mathrm{H}), 2.96(\mathrm{~m}, 1 \mathrm{H}), 2.77(\mathrm{dd}$, $J=4.5,3.9 \mathrm{~Hz}, 1 \mathrm{H}), 2.51(\mathrm{dd}, J=4.9,2.6 \mathrm{~Hz}, 1 \mathrm{H}), 2.49-2.40(\mathrm{~m}, 2 \mathrm{H}), 1.80(\mathrm{~m}, 1 \mathrm{H}), 1.71-1.62(\mathrm{~m}, 2 \mathrm{H})$, $1.37(\mathrm{t}, J=7.0 \mathrm{~Hz}, 3 \mathrm{H}) ;{ }^{13} \mathrm{C}$ NMR $\left(100 \mathrm{MHz}, \mathrm{CDCl}_{3}\right) \delta 161.8(\mathrm{~s}), 161.6(\mathrm{~s}), 143.6(\mathrm{~d}), 141.2(\mathrm{~d}), 134.5$ $(\mathrm{s}), 116.8(\mathrm{~d}), 61.6(\mathrm{t}), 51.8(\mathrm{~d}), 47.4(\mathrm{t}), 31.6(\mathrm{t}), 29.6(\mathrm{t}), 14.6(\mathrm{q}) ; \mathrm{MS}(\mathrm{EI}, 70 \mathrm{eV}): \mathrm{m} / \mathrm{z}$ (relative

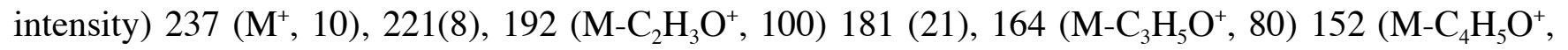
40) 135 (20), 79(20); HRMS (ESI): calcd for $\mathrm{C}_{12} \mathrm{H}_{15} \mathrm{O}_{4} \mathrm{~N}[\mathrm{M}+\mathrm{Na}]^{+}:$260.0893, found: 260.0886 .

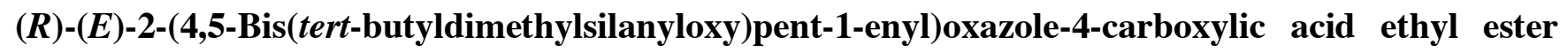
4g.

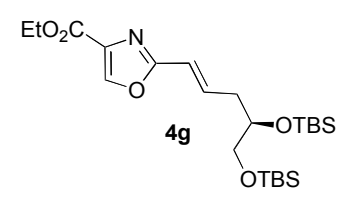


$[\alpha]^{20}{ }_{\mathrm{D}}-10.2\left(c\right.$ 1.85, $\left.\mathrm{CHCl}_{3}\right)$; IR: $v=2955,2930,1748,1723,1113,835 ;{ }^{1} \mathrm{H} \mathrm{NMR}\left(400 \mathrm{MHz}, \mathrm{CDCl}_{3}\right)$ $\delta 8.11(\mathrm{~s}, 1 \mathrm{H}) 6.83(\mathrm{~m}, 1 \mathrm{H}) 6.35(\mathrm{~d}, J=16 \mathrm{~Hz}, 1 \mathrm{H}), 4.38(\mathrm{q}, J=7.0 \mathrm{~Hz}, 2 \mathrm{H}), 3.79(\mathrm{~m}, 1 \mathrm{H}) 3.54(\mathrm{dd}$, $J=9.8,5.0,1 \mathrm{H}), 3.42(\mathrm{dd}, J=9.8,7.0 \mathrm{~Hz}, 1 \mathrm{H}), 2.56(\mathrm{~m}, 1 \mathrm{H}), 2.38(\mathrm{~m}, 1 \mathrm{H}), 1.38,1.36(2 \mathrm{~s}, 18 \mathrm{H}), 0.88$, 0.86, 0.05, $\left.0.04(4 \mathrm{~s}, 12 \mathrm{H}) ;{ }^{13} \mathrm{C} \mathrm{NMR} \mathrm{(100} \mathrm{MHz,} \mathrm{CDCl}_{3}\right) \delta 162.0$ (s), 161.7 (s), 143.4 (d), 139.3 (d), $134.5(\mathrm{~d}), 118.3(\mathrm{~d}), 72.6(\mathrm{~d}), 67.0(\mathrm{t}), 61.5(\mathrm{t}), 38.3(\mathrm{t}), 26.2(\mathrm{q}), 26.1(\mathrm{q}), 18.6(\mathrm{~s}), 18.4(\mathrm{~s}), 14.6(\mathrm{q})$, -4.1 (q), -4.4 (q), -5.0 (q), -5.1 (q); MS (EI, $70 \mathrm{eV}): \mathrm{m} / \mathrm{z}$ (relative intensity) $470\left(\mathrm{M}^{+}, 1\right), 454(4), 412$ $\left(\mathrm{M}-\mathrm{C}_{2} \mathrm{H}_{5} \mathrm{O}^{+}, 100\right), 384(5), 289$ (12), 252 (90), 147 (62), 73 (45); HRMS (ESI): calcd for $\mathrm{C}_{23} \mathrm{H}_{43} \mathrm{O}_{5} \mathrm{NSi}_{2}$ $[\mathrm{M}+\mathrm{Na}]^{+}:$492.2572, found: 492.2563

\section{2-[(4S,5R,E)-6-(tert-Butyldimethylsilanyloxy)-4-(methoxymethoxy)-5-methylhex-1-enyl)oxazole-4-} carboxylic acid ethyl ester $4 \mathrm{~h}$.

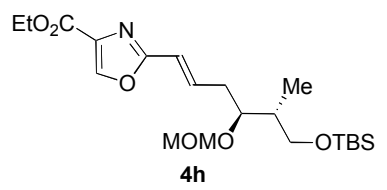

$[\alpha]_{\mathrm{D}}^{20}+11.0\left(c\right.$ 1.3, $\left.\mathrm{CHCl}_{3}\right) ; \mathrm{IR}: v=2927,2856,1746,1722,1315,1146,1111,1036,1036 ;{ }^{1} \mathrm{H}$ NMR $\left(400 \mathrm{MHz}, \mathrm{CDCl}_{3}\right) \delta 8.12(\mathrm{~s}, 1 \mathrm{H}), 6.90(\mathrm{td}, J=6.5,4.02 \mathrm{~Hz}, 1 \mathrm{H}), 6.39\left(\mathrm{~d}_{\text {app }}, J=16.0 \mathrm{~Hz}, 1 \mathrm{H}\right), 4.65(\mathrm{~d}$, $J=6.8 \mathrm{~Hz}, 1 \mathrm{H}), 4.63(\mathrm{~d}, J=6.8 \mathrm{~Hz}, 1 \mathrm{H}), 4.37(\mathrm{q}, J=7 \mathrm{~Hz}, 2 \mathrm{H}), 3.73(\mathrm{~m}, 1 \mathrm{H}), 3.63-3.2 .53(\mathrm{~m}, 2 \mathrm{H})$, $3.35(\mathrm{~s}, 3 \mathrm{H}), 2.58(\mathrm{~m}, 1 \mathrm{H}), 1.90(\mathrm{~m}, 1 \mathrm{H}), 1.40(\mathrm{t}, J=7 \mathrm{~Hz}, 3 \mathrm{H}), 0.90(\mathrm{~d}, J=7.0 \mathrm{~Hz}, 3 \mathrm{H}), 0.89(\mathrm{~s}, 9 \mathrm{H})$, $0.04(\mathrm{~s}, 6 \mathrm{H}) ;{ }^{13} \mathrm{C}$ NMR (100 MHz, $\left.\mathrm{CDCl}_{3}\right) \delta 161.9$ (s), 161.7 (s), 143.3 (d), 139.4 (d), 134.5 (s), 118.1 $(d), 96.8(\mathrm{t}), 78.6(\mathrm{~d}), 65.0(\mathrm{t}), 61.5(\mathrm{t}), 56.1(\mathrm{q}), 39.3(\mathrm{~d}), 35.1(\mathrm{t}), 26.2(\mathrm{q}), 18.6(\mathrm{~s}), 14.6(\mathrm{q}), 13.0(\mathrm{q})$,

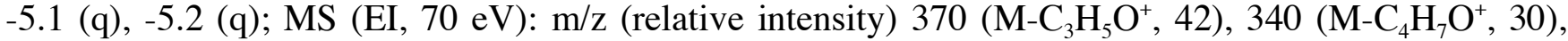
308 (51), 225 (60), 145 (100), 89 (88); HRMS (ESI): calcd for $\mathrm{C}_{21} \mathrm{H}_{37} \mathrm{O}_{6} \mathrm{NSi}[\mathrm{M}+\mathrm{Na}]^{+}:$450.2282, found: 450.2272 . 
(E)-2-[2-(4-Fluorophenyl)vinyl]oxazole-4-carboxylic acid ethyl ester 4i.

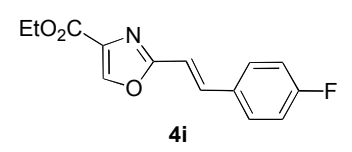

$\mathrm{mp}=126^{\circ} \mathrm{C} ; \mathrm{IR}: v=3084,2976,2927,2356,2118,1714,1506,1314,1159,1112,821,731 ;{ }^{1} \mathrm{H}$ NMR $\left(400 \mathrm{MHz}, \mathrm{CDCl}_{3}\right) \delta 8.19(\mathrm{~s}, 1 \mathrm{H}), 7.58(\mathrm{~d}, J=16.3 \mathrm{~Hz}, 1 \mathrm{H}), 7.53-7.47(\mathrm{~m}, 2 \mathrm{H}), 7.12-7.05(\mathrm{~m}, 2 \mathrm{H}), 6.86$ $(\mathrm{d}, J=13.7 \mathrm{~Hz}, 1 \mathrm{H}), 4.41(\mathrm{q}, J=7.0 \mathrm{~Hz}, 2 \mathrm{H}), 1.40(\mathrm{t}, J=7.0 \mathrm{~Hz}, 3 \mathrm{H}) ;{ }^{13} \mathrm{C} \mathrm{NMR}(100 \mathrm{MHz}$ $\left.\mathrm{CDCl}_{3}\right) \delta 163.9\left(\mathrm{~s}, J_{\mathrm{CF}}^{1}=249 \mathrm{~Hz}\right), 162.3(\mathrm{~s}), 161.6(\mathrm{~s}), 143.6(\mathrm{~d}), 137.3(\mathrm{~d}), 135.1(\mathrm{~d}), 131.6(\mathrm{~s}$, $\left.J_{\mathrm{CF}}^{4}=4 \mathrm{~Hz}\right), 129.5\left(\mathrm{~d}, J_{\mathrm{CF}}^{3}=8 \mathrm{~Hz}\right), 116.4\left(\mathrm{~d}, J^{2}{ }_{\mathrm{CF}}=22 \mathrm{~Hz}\right), 113.1\left(\mathrm{~d}, J_{\mathrm{CF}}^{5}=2 \mathrm{~Hz}\right), 61.6(\mathrm{t}), 14.7(\mathrm{q})$ MS (EI, $70 \mathrm{eV}): \mathrm{m} / \mathrm{z}$ (relative intensity) $262\left(\mathrm{M}+1^{+}, 8\right), 261\left(\mathrm{M}^{+}, 56\right), 260\left(\mathrm{M}-1^{+}, 100\right), 232\left(\mathrm{M}^{-} \mathrm{C}_{2} \mathrm{H}_{5}^{+}\right.$, 53), 216 (10), 187 (15); 159 (27) 149 (55), 133 (40); HRMS (ESI): calcd for $\mathrm{C}_{14} \mathrm{H}_{12} \mathrm{O}_{3} \mathrm{NF}[\mathrm{M}+\mathrm{Na}]^{+}$: 284.0693, found: 284.0687.

(E)-2-(Styryl)oxazole-4-carboxylic acid ethyl ester $\mathbf{4 j}$.

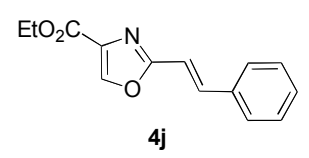

IR: $v=3115,3068,2981,1722,1323,1148,1110,755 ;{ }^{1} \mathrm{H} \mathrm{NMR}\left(400 \mathrm{MHz}, \mathrm{CDCl}_{3}\right) \delta 8.20(\mathrm{~s}, 1 \mathrm{H})$, $7.62(\mathrm{~d}, J=16.3 \mathrm{~Hz}, 1 \mathrm{H}), 7.55-7.50(\mathrm{~m}, 2 \mathrm{H}), 7.43-7.30(\mathrm{~m}, 3 \mathrm{H}), 6.96(\mathrm{~d}, J=16.3 \mathrm{~Hz}, 1 \mathrm{H}), 4.41(\mathrm{q}$, $J=7.0 \mathrm{~Hz}, 2 \mathrm{H}), 1.40(\mathrm{t}, J=7.0 \mathrm{~Hz}, 3 \mathrm{H}) ;{ }^{13} \mathrm{C} \mathrm{NMR}\left(100 \mathrm{MHz}, \mathrm{CDCl}_{3}\right) \delta 162.5(\mathrm{~s}), 161.6(\mathrm{~s}), 143.6(\mathrm{~d})$, 138.6 (d), 135.3 (d), 135.0 (d), 130.0 (d), 129.3 (d), 127.7 (d), 113.3 (d), 61.6 (t), 14.7 (q); MS (EI, 70

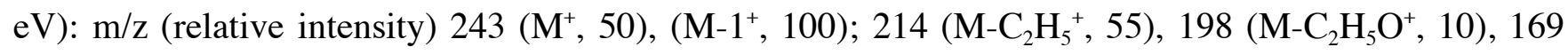
(15), $141(25), 131(33), 115(33), 103(20)$.

(E)-2-[2-(4-Methoxyphenyl)vinyl]oxazole-4-carboxylic acid ethyl ester $4 \mathrm{k}$.

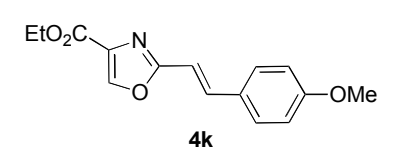


IR: $v=2923,1739,1605,1510,1251,1174,1113 ;{ }^{1} \mathrm{H}$ NMR $\left(400 \mathrm{MHz}, \mathrm{CDCl}_{3}\right) \delta 8.17(\mathrm{~s}, 1 \mathrm{H}), 7.57(\mathrm{~d}$, $J=16.3 \mathrm{~Hz}, 1 \mathrm{H}), 7.49-7.46(\mathrm{~m}, 2 \mathrm{H}), 6.95-6.89(\mathrm{~m}, 2 \mathrm{H}), 6.82(\mathrm{~d}, J=16.3 \mathrm{~Hz}), 4.41(\mathrm{q}, J=7.0 \mathrm{~Hz}, 2 \mathrm{H})$, $3.84(\mathrm{~s}, 1 \mathrm{H}), 1.40(\mathrm{t}, J=7.3 \mathrm{~Hz}, 3 \mathrm{H}) ;{ }^{13} \mathrm{C} \mathrm{NMR}\left(100 \mathrm{MHz}, \mathrm{CDCl}_{3}\right) \delta 162.9(\mathrm{~s}), 161.7(\mathrm{~s}), 161.3(\mathrm{~s})$, 143.4 (d), 138.2 (d), 135.0 (s), 129.3 (d), 128.2 (s), 114.8 (d), $111.01(\mathrm{~d}), 61.6$ (t), 55.7 (q), 14.7 (q); MS (EI, $70 \mathrm{eV}): \mathrm{m} / \mathrm{z}$ (relative intensity) $273\left(\mathrm{M}^{+}, 95\right), 272(\mathrm{M}-1,100), 244\left(\mathrm{M}-\mathrm{C}_{2} \mathrm{H}_{5}^{+}, 40\right), 199(13), 171$ (20), 161 (80), 145 (20), 102 (18); HRMS (ESI): calcd for $\mathrm{C}_{15} \mathrm{H}_{15} \mathrm{O}_{4} \mathrm{~N}[\mathrm{M}+\mathrm{Na}]^{+}$: 296.0893, found: 296.0887.

(E)-2-(3-Oxopent-1-enyl)oxazole-4-carboxylic acid ethyl ester 41.

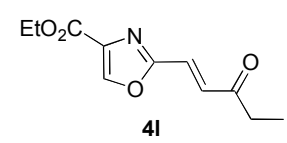

$\mathrm{mp}=125^{\circ} \mathrm{C} ; \mathrm{IR}: v=3125,3069,2984,1721,1668,1328 ;{ }^{1} \mathrm{H} \mathrm{NMR}\left(400 \mathrm{MHz}, \mathrm{CDCl}_{3}\right) \delta 8.25(\mathrm{~s}, 1 \mathrm{H})$, $7.30(\mathrm{~d}, J=16.0 \mathrm{~Hz}, 1 \mathrm{H}), 7.11(\mathrm{~d}, J=16.0 \mathrm{~Hz}, 1 \mathrm{H}), 4.40(\mathrm{q}, J=7.0 \mathrm{~Hz}, 2 \mathrm{H}), 2.68(\mathrm{q}, J=7.3 \mathrm{~Hz}, 2 \mathrm{H})$, $1.38(\mathrm{t}, J=7.3 \mathrm{~Hz}, 3 \mathrm{H}), 1.15(\mathrm{t}, J=7.3 \mathrm{~Hz}, 3 \mathrm{H}) ;{ }^{13} \mathrm{C} \mathrm{NMR}\left(100 \mathrm{MHz}, \mathrm{CDCl}_{3}\right) \delta 199.8(\mathrm{~s}), 161.0(\mathrm{~s})$, 160. 4 (s), 145.0 (d), 135.9 (d), 133.5 (d), 125.3 (d), 61.9 (t), 35.2 (t), 14.6 (q), 8.1 (q); MS (EI, 70 eV): m/z (relative intensity) $223\left(\mathrm{M}^{+}, 30\right), 194\left(\mathrm{M}-\mathrm{C}_{2} \mathrm{H}_{5}^{+}, 100\right), 166\left(\mathrm{M}-\mathrm{C}_{3} \mathrm{H}_{5} \mathrm{O}^{+}, 27\right) 147$ (24), 138 (36); HRMS (ESI): calcd for $\mathrm{C}_{11} \mathrm{H}_{13} \mathrm{O}_{4} \mathrm{~N}[\mathrm{M}+\mathrm{Na}]^{+}:$246.0737, found: 246.0731 .

(E)-2-[(2-Methoxycarbonyl)vinyl]oxazole-4-carboxylic acid ethyl ester 4m.

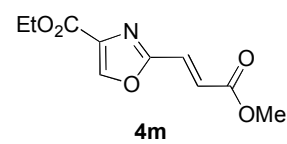

$\mathrm{mp}=121{ }^{\circ} \mathrm{C} ; \mathrm{IR}: v=3128,2917,2850,1715,1330,1214,1145 ;{ }^{1} \mathrm{H} \mathrm{NMR}\left(400 \mathrm{MHz}, \mathrm{CDCl}_{3}\right) \delta 8.25$, $(\mathrm{s}, 1 \mathrm{H}), 7.45(\mathrm{~d}, J=16.0,1 \mathrm{H}), 6.86(\mathrm{~d}, J=16.0 \mathrm{~Hz}, 1 \mathrm{H}), 4.41(\mathrm{q}, J=6.6 \mathrm{~Hz}, 2 \mathrm{H}), 3.83(\mathrm{~s}, 3 \mathrm{H}), 1.39(\mathrm{t}$, $J=7.3 \mathrm{~Hz}, 3 \mathrm{H}) ;{ }^{13} \mathrm{C} \mathrm{NMR}\left(100 \mathrm{MHz}, \mathrm{CDCl}_{3}\right) \delta 164.7$ (s), 161.1 (s), 160.2 (s), 144.8 (d), 135.7 (s), 129.7 (d), 127.2 (d), 61.9 (t), 28.4 (q), 14.6 (q); MS (EI, 70 eV): m/z (relative intensity) $226\left(\mathrm{M}+1^{+}, 1\right)$, 


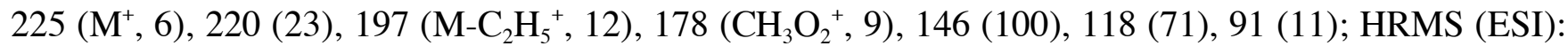

calcd for $\mathrm{C}_{10} \mathrm{H}_{11} \mathrm{O}_{5} \mathrm{~N}[\mathrm{M}+\mathrm{Na}]^{+}: 248.0529$, found: 248.0524 .

\section{(E)-2-[(2-tert-Butoxycarbonyl)vinyl]oxazole-4-carboxylic acid ethyl ester 4 n.}

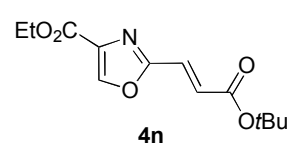

$\mathrm{mp}=71{ }^{\circ} \mathrm{C} ; \mathrm{IR}: v=2980,1746,1715,1311,1154 ;{ }^{1} \mathrm{H} \mathrm{NMR}\left(400 \mathrm{MHz}, \mathrm{CDCl}_{3}\right) \delta 8.23(\mathrm{~s}, 1 \mathrm{H}), 7.37(\mathrm{~d}$, $J=16 \mathrm{~Hz}, 1 \mathrm{H}), 6.76(\mathrm{~d}, J=16.1 \mathrm{~Hz}, 1 \mathrm{H}), 4.41(\mathrm{q}, J=7.3 \mathrm{~Hz}, 2 \mathrm{H}), 1.52(\mathrm{~s}, 9 \mathrm{H}), 1.39(\mathrm{t}, J=7.3 \mathrm{~Hz}$, $3 \mathrm{H}) ;{ }^{13} \mathrm{C} \mathrm{NMR}\left(100 \mathrm{MHz}, \mathrm{CDCl}_{3}\right) \delta 166.0(\mathrm{~s}), 161.1(\mathrm{~s}), 160.0(\mathrm{~s}), 144.9(\mathrm{~d}), 135.8(\mathrm{~s}), 128.3(\mathrm{~d}), 127.2$ (d), 61.9 (t), 52.7 (q), 30.0 (s), 14.6 (q); MS (EI, 70 eV): m/z (relative intensity) $223\left(\mathrm{M}^{+}, 21\right), 222$

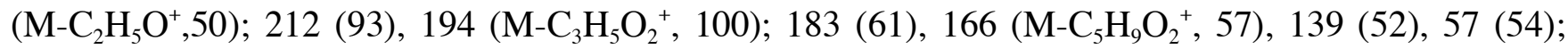
HRMS (ESI): calcd for $\mathrm{C}_{13} \mathrm{H}_{17} \mathrm{O}_{5} \mathrm{~N}[\mathrm{M}+\mathrm{Na}]^{+}:$290.0999, found: 290.0994 .

\section{2-Phenyl-4-trifluoromethanesulfonyloxy oxazole ${ }^{1} 9$.}
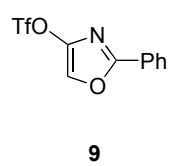

To a solution of benzamide $(200 \mathrm{mg}, 1.65 \mathrm{mmol})$ in $\mathrm{CH}_{2} \mathrm{Cl}_{2}(16 \mathrm{~mL})$ was added oxalyl chloride $(0.216$ $\mathrm{mL}, 2.48 \mathrm{mmol}, 1.5$ equiv) and the resulting was heated at reflux for $16 \mathrm{~h}$. Upon cooling to $\mathrm{rt}$, the crude benzoyl isocyanate was concentrated in vacuo under moisture-free conditions, diluted with $\mathrm{MeCN}$ $(11 \mathrm{~mL})$, cooled to $0^{\circ} \mathrm{C}$, and $\mathrm{TMSCH}_{2} \mathrm{~N}_{2}\left(\left[2.0 \mathrm{M}\right.\right.$ in $\left.\mathrm{Et}_{2} \mathrm{O}\right], 0.991 \mathrm{~mL}, 1.98 \mathrm{mmol}, 1.2$ equiv) was added dropwise. The cold bath was removed and the mixture reached rt over a 45 min period. The crude residue was diluted with $\mathrm{CH}_{2} \mathrm{Cl}_{2}(32 \mathrm{~mL})$ and cooled to $-78{ }^{\circ} \mathrm{C}$ to which was successively added $\mathrm{Et}_{3} \mathrm{~N}$

[1] Flegeau, E. F.; Popkin, M. E.; Greaney, M. F. Org. Lett. 2006, 8, 2495-2498. 
(0.46 mL, $3.30 \mathrm{mmol}, 2$ equiv) and triflic anhydride $(0.42 \mathrm{~mL}, 2.6 \mathrm{mmol}, 1.6$ equiv). The cold bath was removed and the content was allowed to reach rt. The solvent was removed in vacuo and the residue was purified by flash chromatography on silica gel (2/98 $\mathrm{Et}_{2} \mathrm{O} / n$-pentane) to give 2-phenyl-4trifluoromethanesulfonyloxy-oxazole $(9)(0.372 \mathrm{~g}, 77 \%)$ as an amorphous white solid $\left(\mathrm{mp}<25^{\circ} \mathrm{C}\right)$. IR: $v=3185,3146,3069,2927,2856,1587,1435,1219,1138,{ }^{1} \mathrm{H}$ NMR $\left(400 \mathrm{MHz}, \mathrm{CDCl}_{3}\right) \delta 8.03-8.00$ $(\mathrm{m}, 2 \mathrm{H}), 7.74(\mathrm{~s}, 1 \mathrm{H}), 7.54-7.46(\mathrm{~m}, 3 \mathrm{H}) ;{ }^{13} \mathrm{C} \mathrm{NMR}\left(100 \mathrm{MHz}, \mathrm{CDCl}_{3}\right) \delta 160.0(\mathrm{~s}), 146.3(\mathrm{~s}), 131.9(\mathrm{~d})$, 129.3 (d), 126.9 (d), 126.6 (d), 126.5 (d), 119.0 (d, $\left.J_{\mathrm{CF}}=319 \mathrm{~Hz}\right) ; \mathrm{MS}$ (EI, $\left.70 \mathrm{eV}\right): \mathrm{m} / \mathrm{z}$ (relative intensity) $171\left(\mathrm{M}^{+}, 100\right), 143\left(\mathrm{M}-\mathrm{C}_{2} \mathrm{H}_{4}^{+}, 60\right)$.

\section{2-phenyl-4-vinyloxazole 2.}

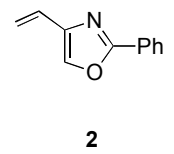

To a solution of 2-phenyloxazol-4-yl trifluoromethanesulfonate (550 mg, $1.88 \mathrm{mmol})$ in degassed 1,4dioxane $(20 \mathrm{~mL})$ was added tri- $n$-butyl vinylstanane $(0.60 \mathrm{~mL}, 2.06 \mathrm{mmol}, 1.1$ equiv), lithium chloride (400 mg, $9.39 \mathrm{mmol}, 5$ equiv), and $\mathrm{Pd}\left(\mathrm{PPh}_{3}\right)_{4}(217 \mathrm{mg}, 0.188 \mathrm{mmol}, 0.1$ equiv). The contents were heated in a sealed tube and heated at $100{ }^{\circ} \mathrm{C}$ for $20 \mathrm{~h}$. After reaching $\mathrm{rt}$ a satd $\mathrm{NH}_{4} \mathrm{Cl}$ solution was added and the organic layer was extracted with EtOAc $(3 \times 15 \mathrm{~mL})$. The organic layers were combined, washed with $\mathrm{H}_{2} \mathrm{O}$, brine, dried over $\mathrm{MgSO}_{4}$, filtered, and contrated in vacuo. The crude residue was then purified by column chromatography on silica gel using a gradient of eluent (0/100: 1/9 $\mathrm{Et}_{2} \mathrm{O} / \mathrm{n}$-pentane) to provide 2-phenyl-4-vinyloxazole $(163 \mathrm{mg}, 51 \%)$ as a colorless oil. ${ }^{1} \mathrm{H} \mathrm{NMR}\left(400 \mathrm{MHz}, \mathrm{CDCl}_{3}\right)$ $\delta 8.10-8.06(\mathrm{~m}, 2 \mathrm{H}), 7.61(\mathrm{~s}, 1 \mathrm{H}), 7.47-7.44(\mathrm{~m}, 3 \mathrm{H}), 6.61(\mathrm{ddd}, J=17.3,10.8,1.5 \mathrm{~Hz}, 1 \mathrm{H}), 6.06(\mathrm{dd}$, $J=17.3,1.5 \mathrm{~Hz}, 1 \mathrm{H}), 5.35(\mathrm{dd}, J=10.8,1.5 \mathrm{~Hz}, 1 \mathrm{H}) ;{ }^{13} \mathrm{C} \mathrm{NMR}\left(100 \mathrm{MHz}, \mathrm{CDCl}_{3}\right) \delta 162.1(\mathrm{~s}), 140.8$ (s), 135.3 (d), 130.8 (d), 129.0 (d); 127.8 (s), 126.9 (d), 125.6 (d), 116.5 (t); MS (EI, 70 eV): m/z (relative intensity) $171\left(\mathrm{M}^{+}, 100\right), 143\left(\mathrm{M}-\mathrm{C}_{2} \mathrm{H}_{4}^{+}, 60\right), 115$ (25), 90 (25), 77 (15); HRMS (ESI): calcd for $\mathrm{C}_{11} \mathrm{H}_{10} \mathrm{ON}^{+}:$172.0757, found: 172.0753 . 
(E)-4-(4-methylpent-1-enyl)-2-phenyloxazole 5a.

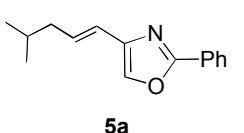

IR: $v=2956,2936,2869,1718,1558,1450,716,691 ;{ }^{1} \mathrm{H}$ NMR $\left(400 \mathrm{MHz}, \mathrm{CDCl}_{3}\right) \delta 8.08-8.04(\mathrm{~m}$, 2H), $7.53(\mathrm{~s}, 1 \mathrm{H}), 7.46-7.43(\mathrm{~m}, 3 \mathrm{H}), 6.53(\mathrm{~m}, 1 \mathrm{H}), 6.25(\mathrm{dt}, J=15.6,1.5 \mathrm{~Hz}, 1 \mathrm{H}), 2.11(\mathrm{td}, J=7.3,1.3$ $\mathrm{Hz}, 2 \mathrm{H}), 1.77$ (septuplet, $J=6.5 \mathrm{~Hz}, 1 \mathrm{H}), 0.96(\mathrm{~d}, J=6.5 \mathrm{~Hz}, 6 \mathrm{H}) ;{ }^{13} \mathrm{C} \mathrm{NMR}\left(100 \mathrm{MHz}, \mathrm{CDCl}_{3}\right) \delta 161.9$ (s), $140.8(\mathrm{~s}), 134.1$ (d), 132.8 (d), 130.6 (d), 129.0 (d), 127.9 (s), 126.9 (d), 119.5 (d), 42.7 (t), 28.7 (d),

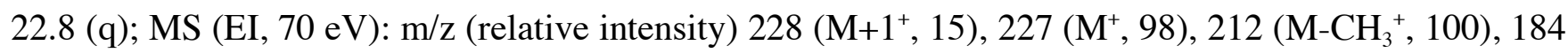

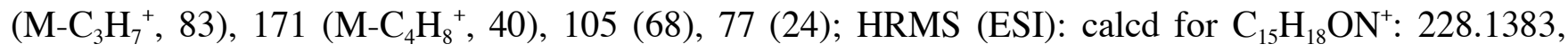
found: 228.1377 .

(E)-2-[3-(2-Phenyloxazol-4-yl)allyl]malonic acid dimethyl ester 5d.

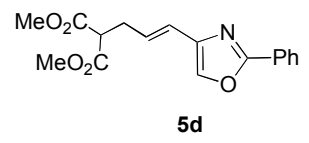

IR: $v=2954,1733,1232,1200,1154,693 ;{ }^{1} \mathrm{H} \mathrm{NMR}\left(400 \mathrm{MHz}, \mathrm{CDCl}_{3}\right) \delta 8.07-8.02(\mathrm{~m}, 2 \mathrm{H}), 7.55(\mathrm{~s}$, 1H), 7.46-7.44 (m, 3H), $6.46(\mathrm{dt}, J=15.3,7.0 \mathrm{~Hz}, 1 \mathrm{H}), 6.36(\mathrm{~d}, J=15.6 \mathrm{~Hz}, 1 \mathrm{H}), 3.75(\mathrm{~s}, 6 \mathrm{H}), 3.56(\mathrm{t}$, $J=7.5 \mathrm{~Hz}, 1 \mathrm{H}), 2.83(\mathrm{td}, J=7.2,0.76 \mathrm{~Hz}, 2 \mathrm{H}) ;{ }^{13} \mathrm{C} \mathrm{NMR}\left(100 \mathrm{MHz}, \mathrm{CDCl}_{3}\right) \delta 169.6(\mathrm{~s}), 162.1(\mathrm{~s})$, 140.0 (s), 134.9 (d) 130.8 (d), 129.1 (d), 128.2 (d), 127.5 (s), 126.9 (d), 121.5 (d), 53.0 (d), 51.8 (d), 32.4 (t); MS (EI, $70 \mathrm{eV}): \mathrm{m} / \mathrm{z}$ (relative intensity) 258 (12), 257 (53), $256\left(\mathrm{M}_{-} \mathrm{C}_{2} \mathrm{H}_{3} \mathrm{O}_{2}^{+}, 60\right), 242$ $\left(\mathrm{M}-\mathrm{C}_{3} \mathrm{H}_{10} \mathrm{O}_{2}^{+}, 65\right), 73$ (100); HRMS (ESI): calcd for $\mathrm{C}_{17} \mathrm{H}_{17} \mathrm{O}_{5} \mathrm{~N}[\mathrm{M}+\mathrm{Na}]^{+}:$338.0999, found: 338.0992 .

\section{(E)-1-(2-Phenyloxazol-4-yl)pent-1-en-3-one 51.}

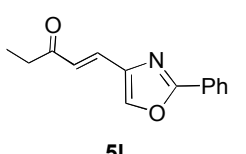


$\mathrm{mp}=82{ }^{\circ} \mathrm{C} ; \mathrm{IR}: v=3144,2943,2902,1693,1622,1573,1107 ;{ }^{1} \mathrm{H} \mathrm{NMR}\left(400 \mathrm{MHz}, \mathrm{CDCl}_{3}\right)$ $\delta$ 8.09-8.06 (m, 2H), $7.86(\mathrm{~s}, 1 \mathrm{H}), 7.49-7.43(\mathrm{~m}, 3 \mathrm{H}), 7.42(\mathrm{~s}, 1 \mathrm{H}), 7.07(\mathrm{dd}, J=15.7,0.5 \mathrm{~Hz}, 1 \mathrm{H}), 2.67$ $(\mathrm{q}, J=7.5 \mathrm{~Hz}, 2 \mathrm{H}), 1.17(\mathrm{t}, J=7.3 \mathrm{~Hz}, 3 \mathrm{H}) ;{ }^{13} \mathrm{C} \mathrm{NMR}\left(100 \mathrm{MHz}, \mathrm{CDCl}_{3}\right) \delta 201.0(\mathrm{~s}), 162.8(\mathrm{~s}), 139.9$ (d), $139.0(\mathrm{~s}), 131.3(\mathrm{~s}), 129.8(\mathrm{~s}), 129.2$ (s), 127.5 (s), 127.1 (s), 127.0 (s), 35.4 (t), 8.4 (q); MS (EI, 70

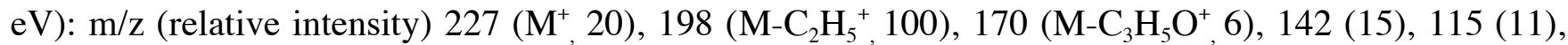
77 (5); HRMS (ESI): calcd for $\mathrm{C}_{14} \mathrm{H}_{13} \mathrm{O}_{2} \mathrm{~N}[\mathrm{M}+\mathrm{Na}]^{+}: 250.0839$, found: 250.0833 .

\section{(E)-3-(2-Phenyloxazol-4-yl)acrylic acid methyl ester 5m.}

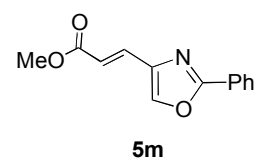

$\mathrm{mp}=114{ }^{\circ} \mathrm{C} ; \mathrm{IR}: \quad v=3155,3046,2954,2923,2849,1713,1645,1163,1109 ;{ }^{1} \mathrm{H} \mathrm{NMR}(400 \mathrm{MHz}$, $\left.\mathrm{CDCl}_{3}\right) \delta 8.08-8.06(\mathrm{~m}, 2 \mathrm{H}), 7.83(\mathrm{~s}, 1 \mathrm{H}), 7.55(\mathrm{~d}, J=15.3 \mathrm{~Hz}, 1 \mathrm{H}), 7.50-7.46(\mathrm{~m}, 3 \mathrm{H}), 6.75(\mathrm{~d}$, $J=15.3 \mathrm{~Hz}, 1 \mathrm{H}), 3.81(\mathrm{~s}, 3 \mathrm{H}) ;{ }^{13} \mathrm{C} \mathrm{NMR}\left(100 \mathrm{MHz}, \mathrm{CDCl}_{3}\right) \delta 167.7(\mathrm{~s}), 162.8(\mathrm{~s}), 139.3(\mathrm{~d}), 138.8$ (s), 132.8 (d), 131.3 (d), 129.2 (d), 127.2 (d), 127.1 (d), 120.3 (d); 52.1 (q); EIMS m/z (relative intensity) $230\left(\mathrm{M}+1^{+}, 15\right), 229\left(\mathrm{M}^{+}, 100\right), 198\left(\mathrm{M}-\mathrm{CH}_{3} \mathrm{O}^{+}, 25\right), 105$ (85); HRMS (ESI): calcd for $\mathrm{C}_{13} \mathrm{H}_{11} \mathrm{O}_{3} \mathrm{~N}$ $[\mathrm{M}+\mathrm{Na}]^{+}:$252.0631, found: 252.0626 . 
III. Copies of ${ }^{1} \mathrm{H}$ NMR \& ${ }^{13} \mathrm{C}$ NMR 

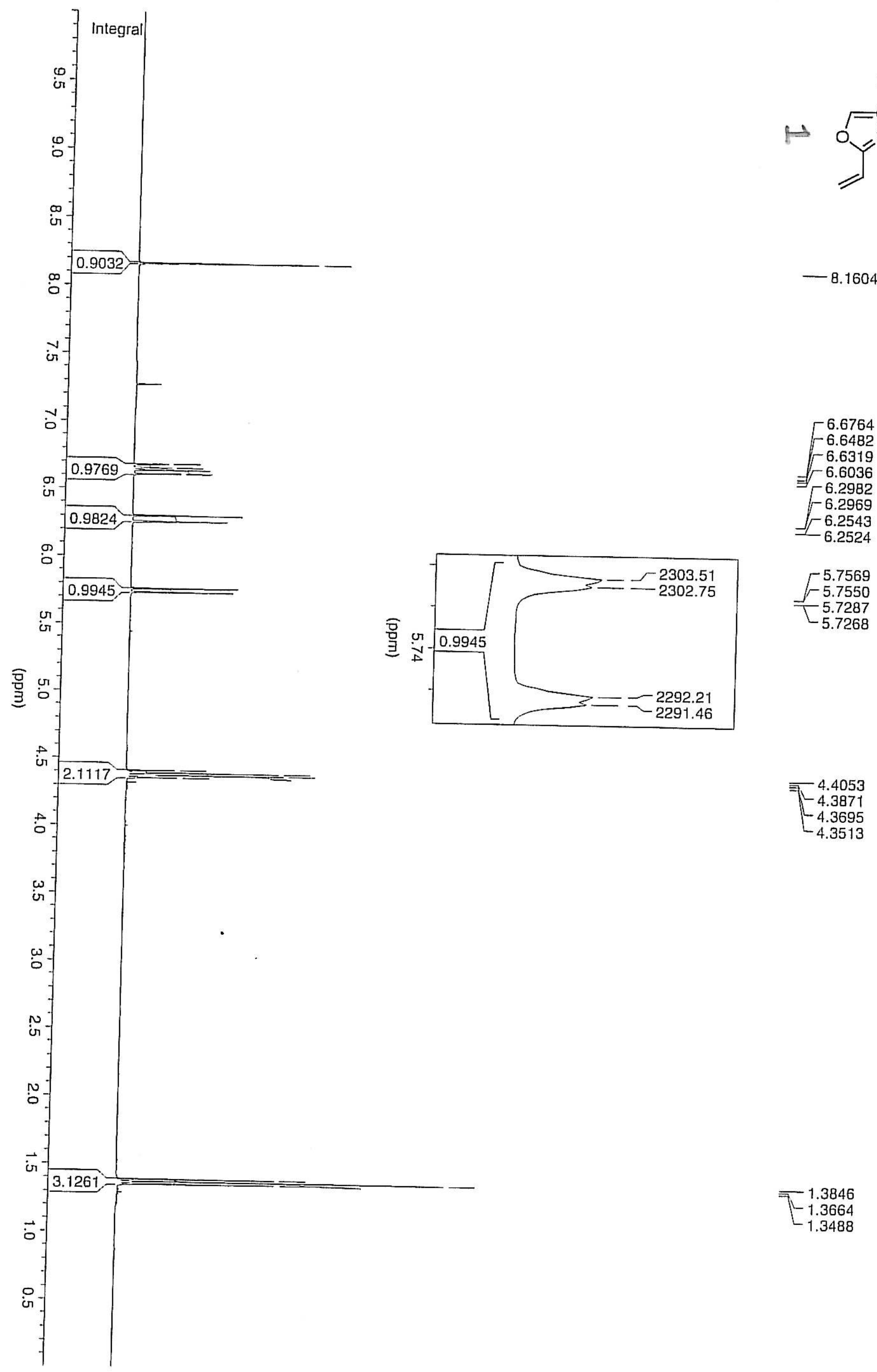

马 4.4053

$-4.3871$

$-4.3695$

$-4.3513$

1.3846

$-1.3664$

$-1.3488$ 

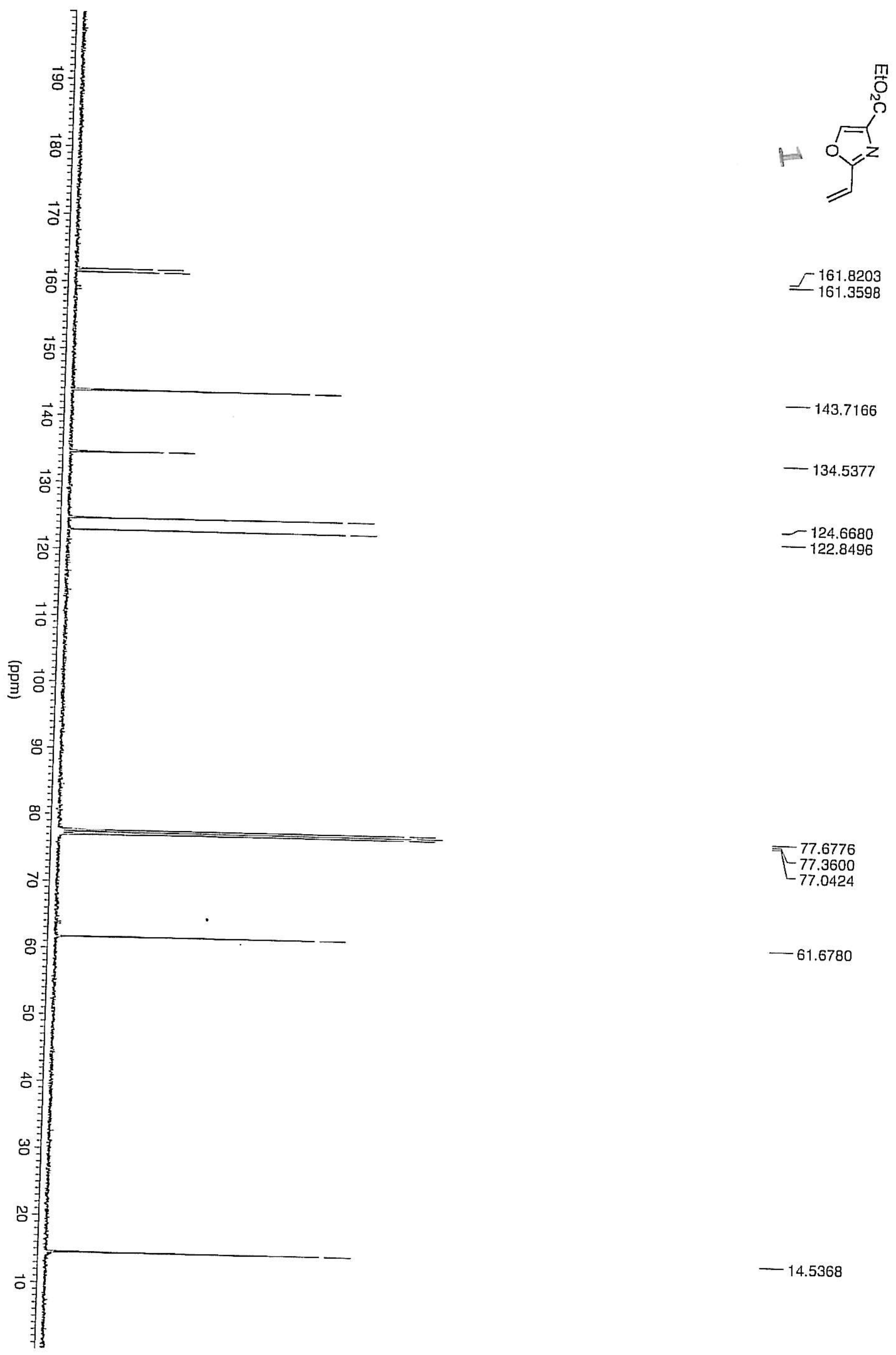

$-134.5377$

$-124.6680$

$-122.8496$

161.8203

161.3598

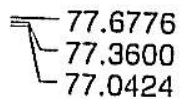

$-61.6780$

$-14.5368$ 


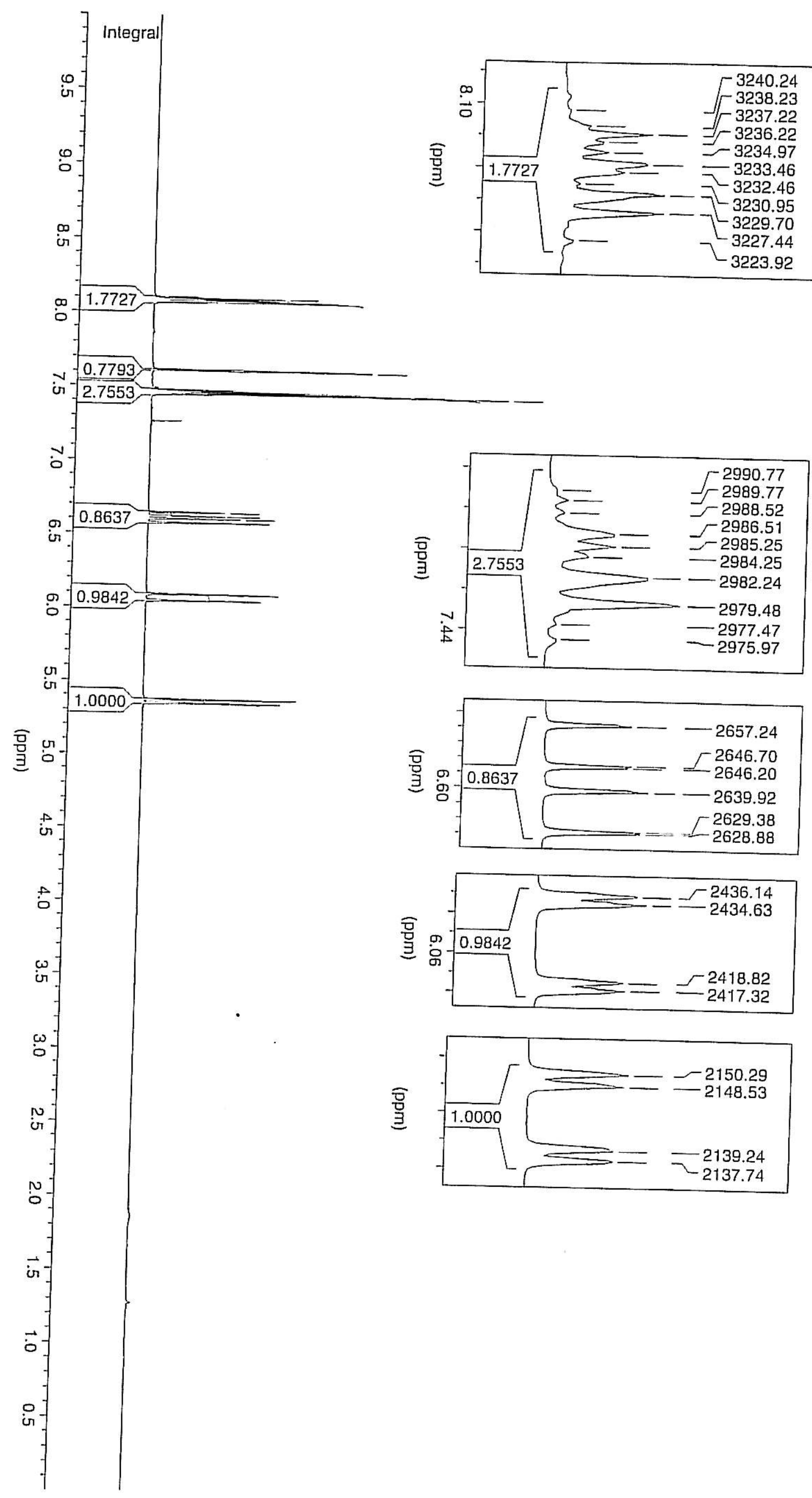




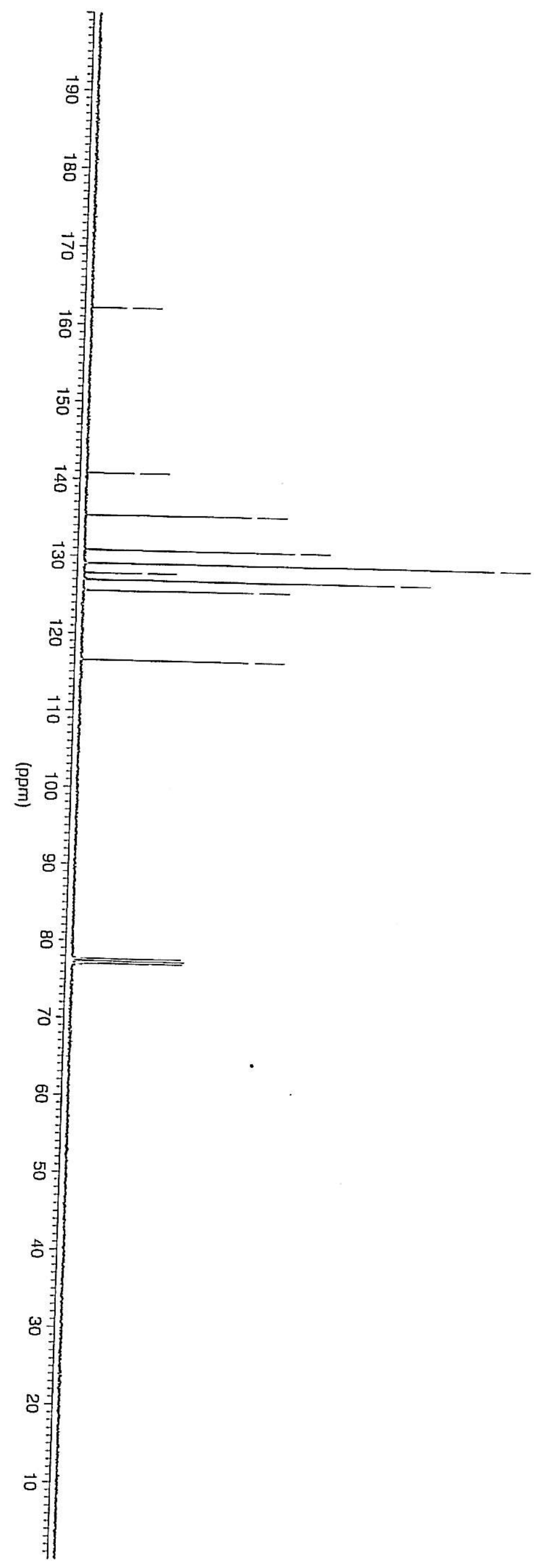

$-140.7867$

135.3079

130.7581

129.0351

- 127.7567

126.9071

$-125.5970$

$-116.4895$ 

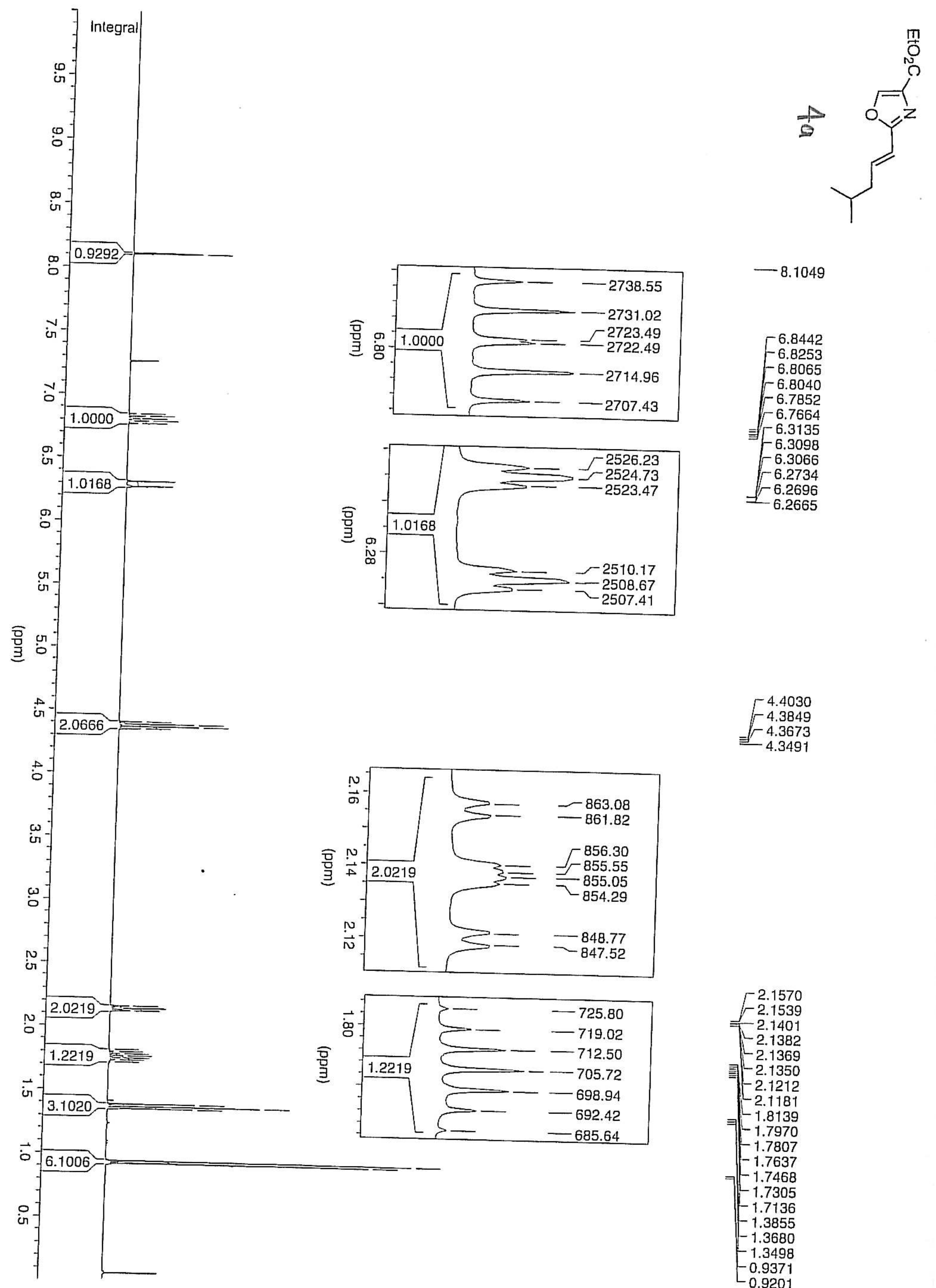

$-8.1049$

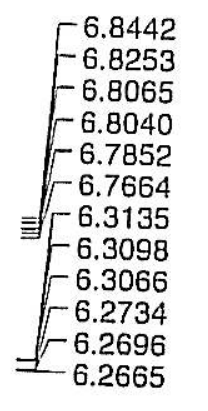

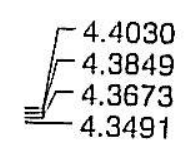

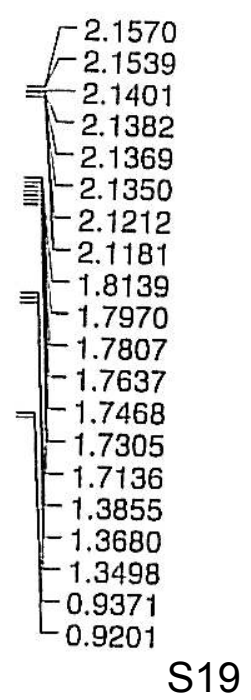




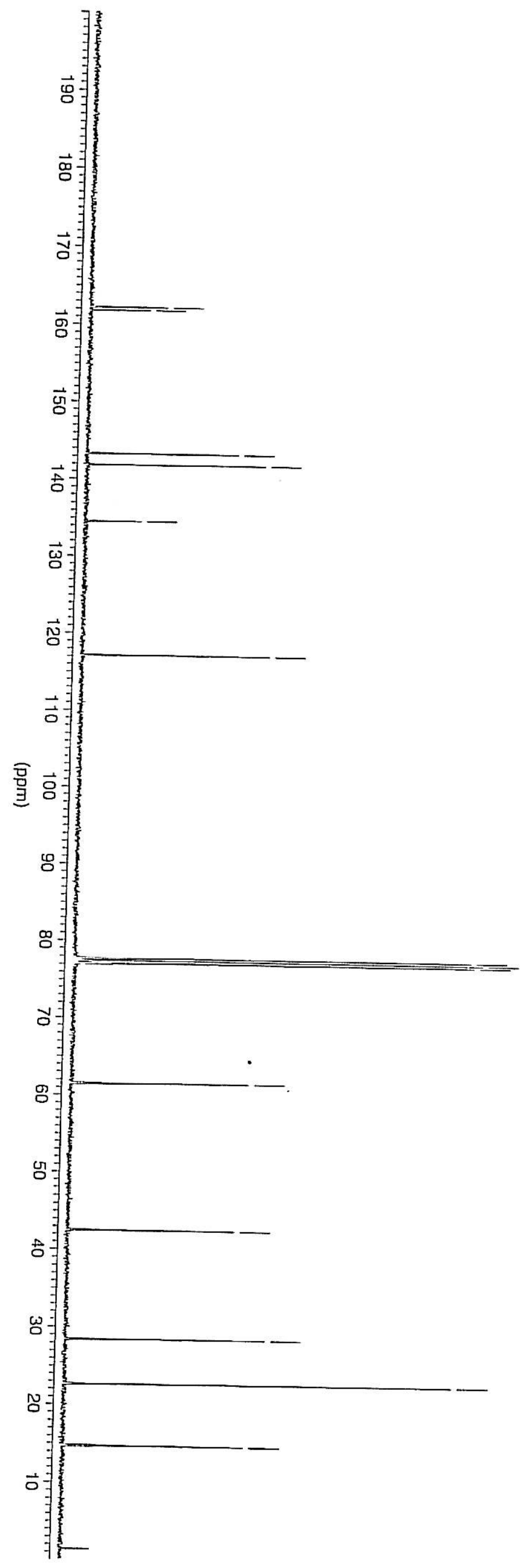

$\Gamma^{162.1300}$

$-143.2164$

141.8268

$-134.4742$

$-117.0691$

$-61.5113$

$-42.4229$

$-28.3766$

$-22.6200$

$-14.6321$ 


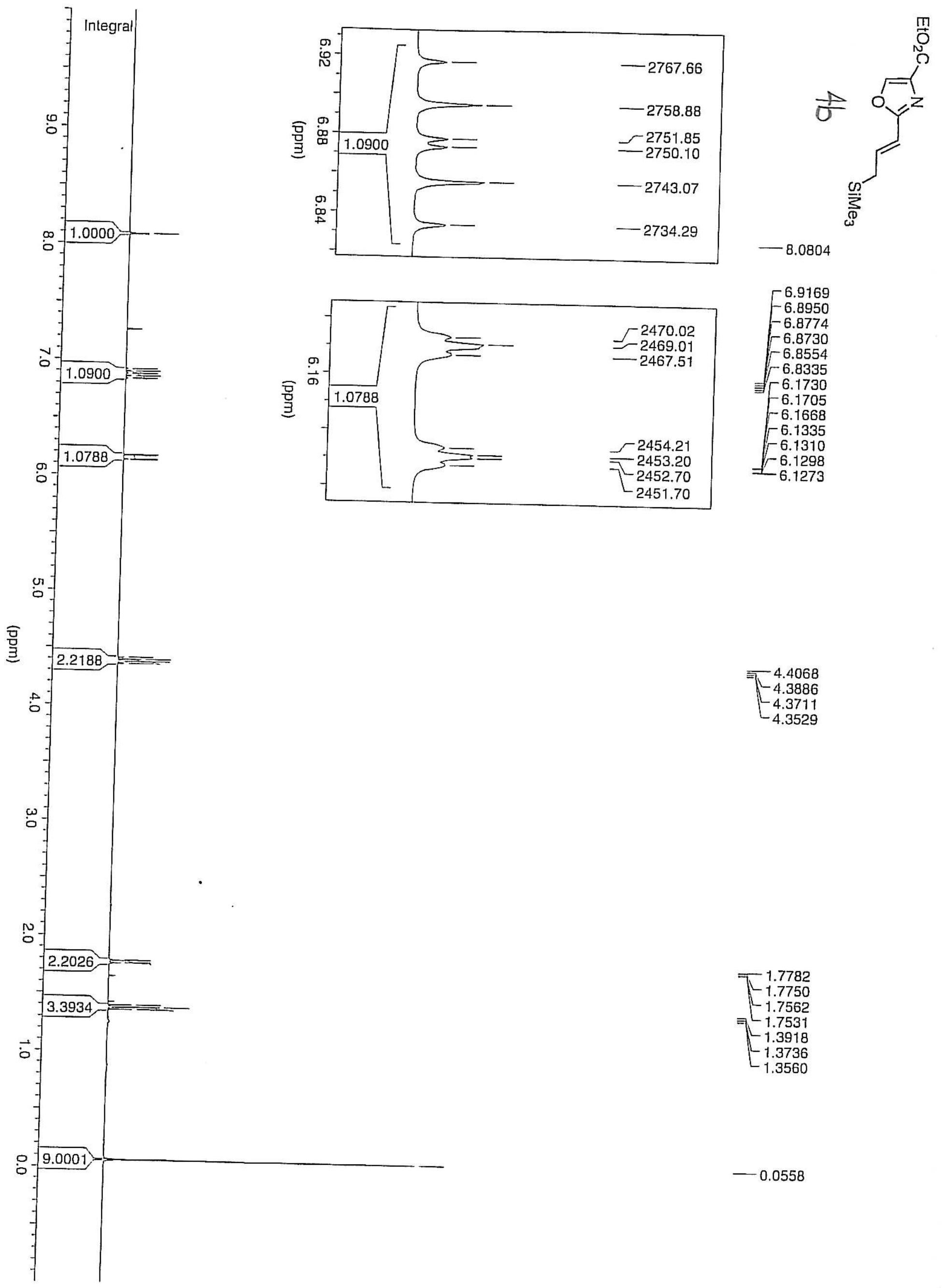



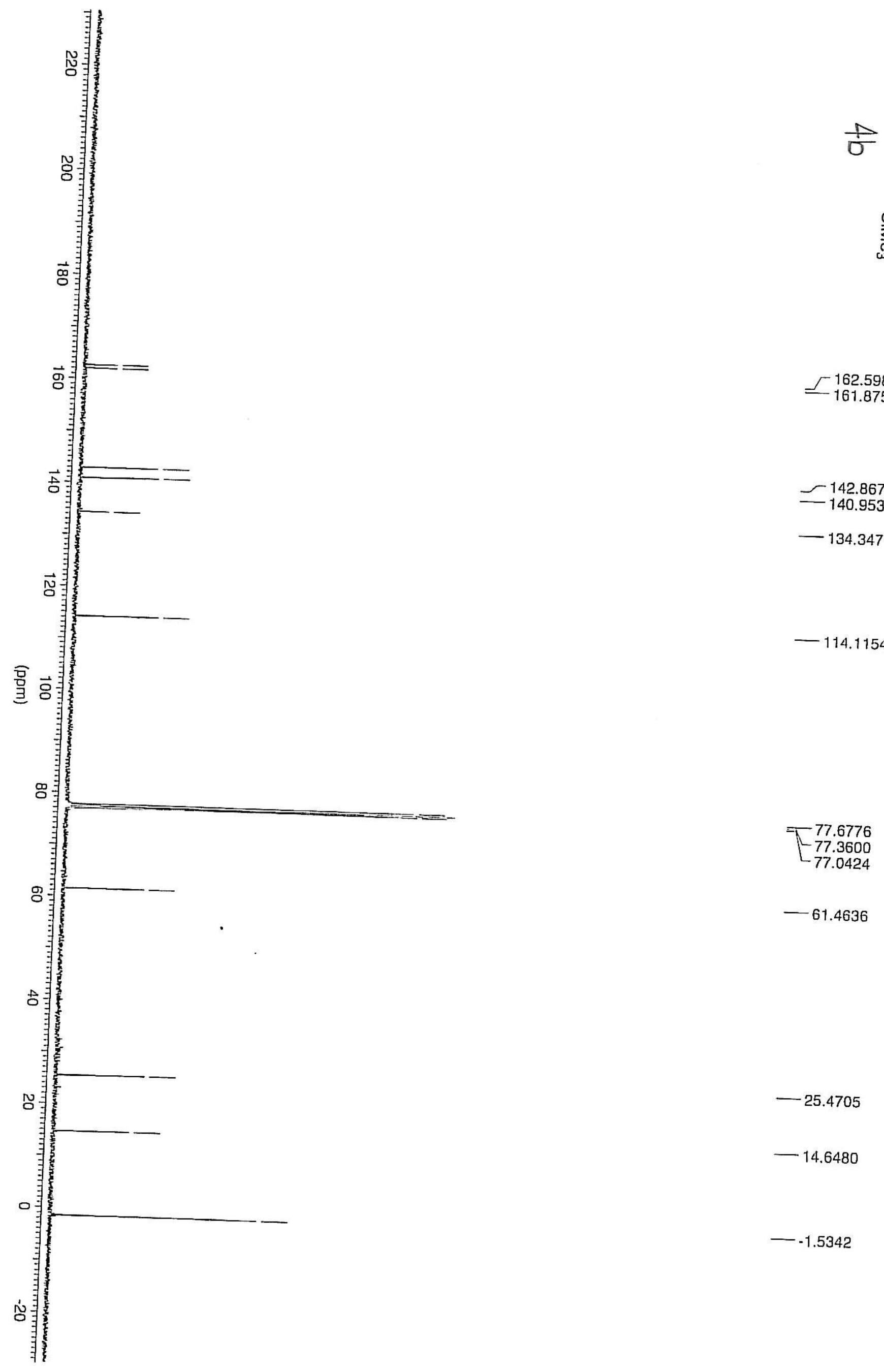

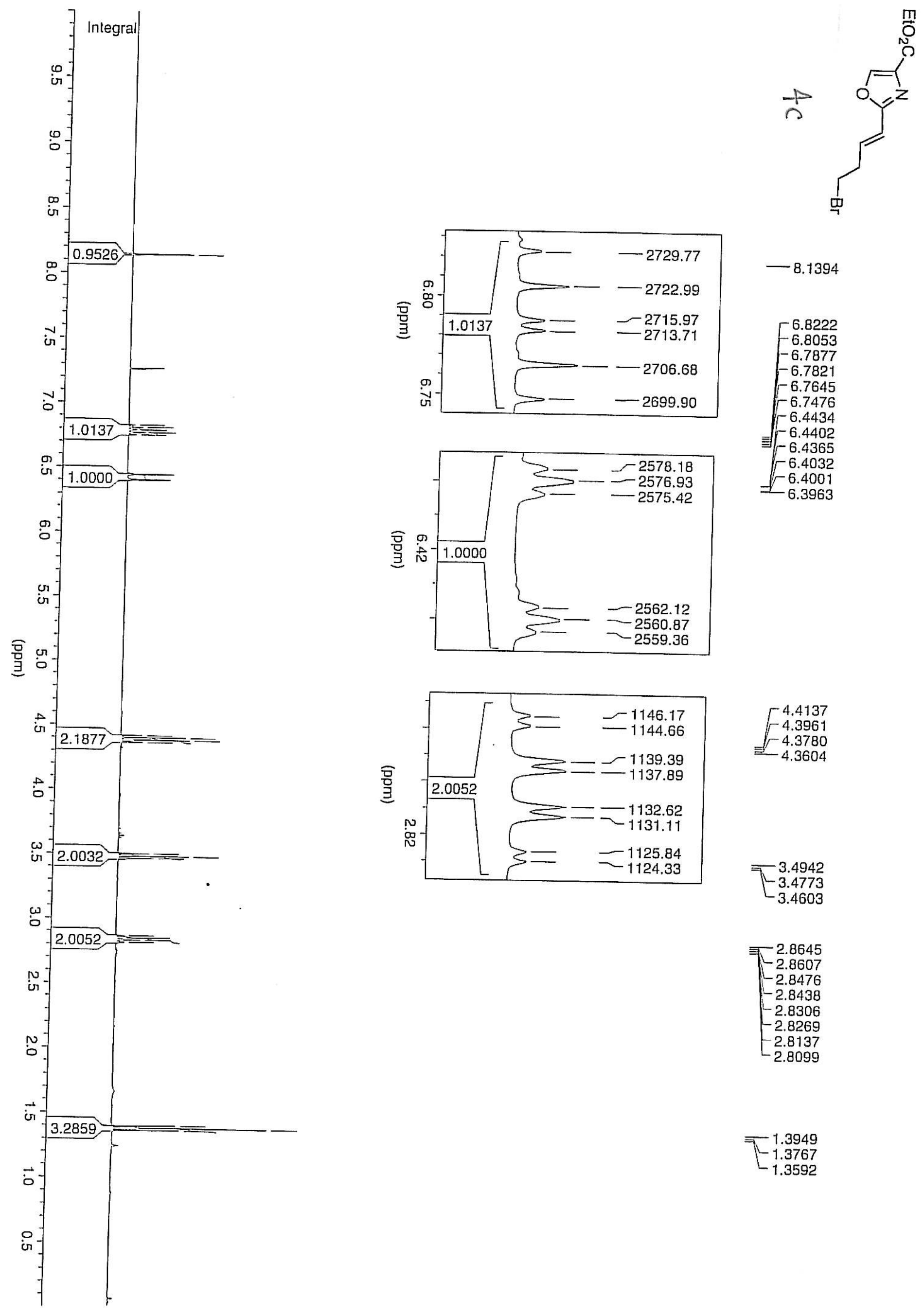

$-8.1394$
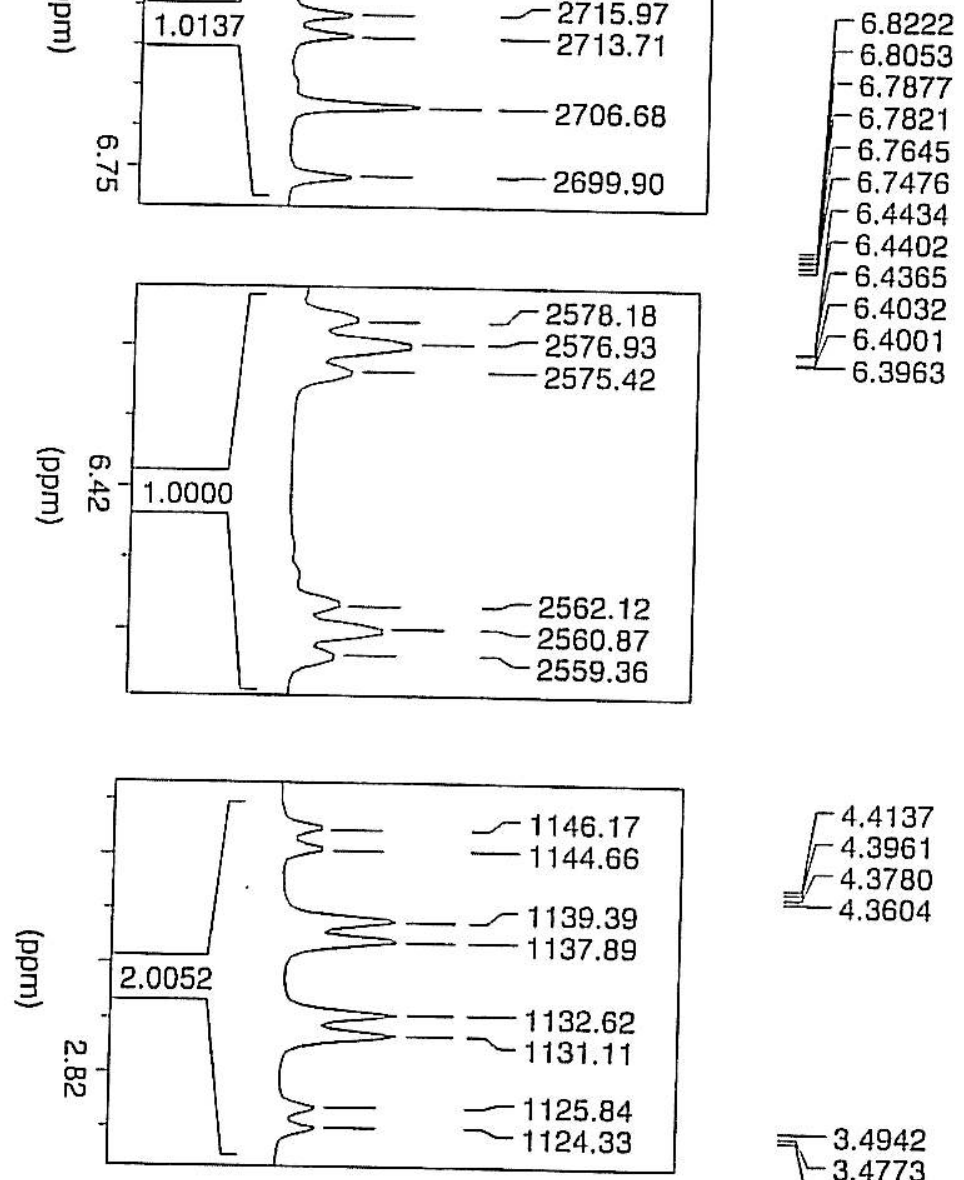

4.4137
-4.3961
-4.3780
$=4.3604$

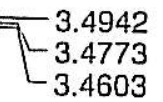

3.4603

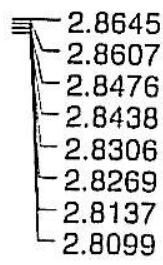

=1.3949

$-1.3767$

$-1.3592$ 


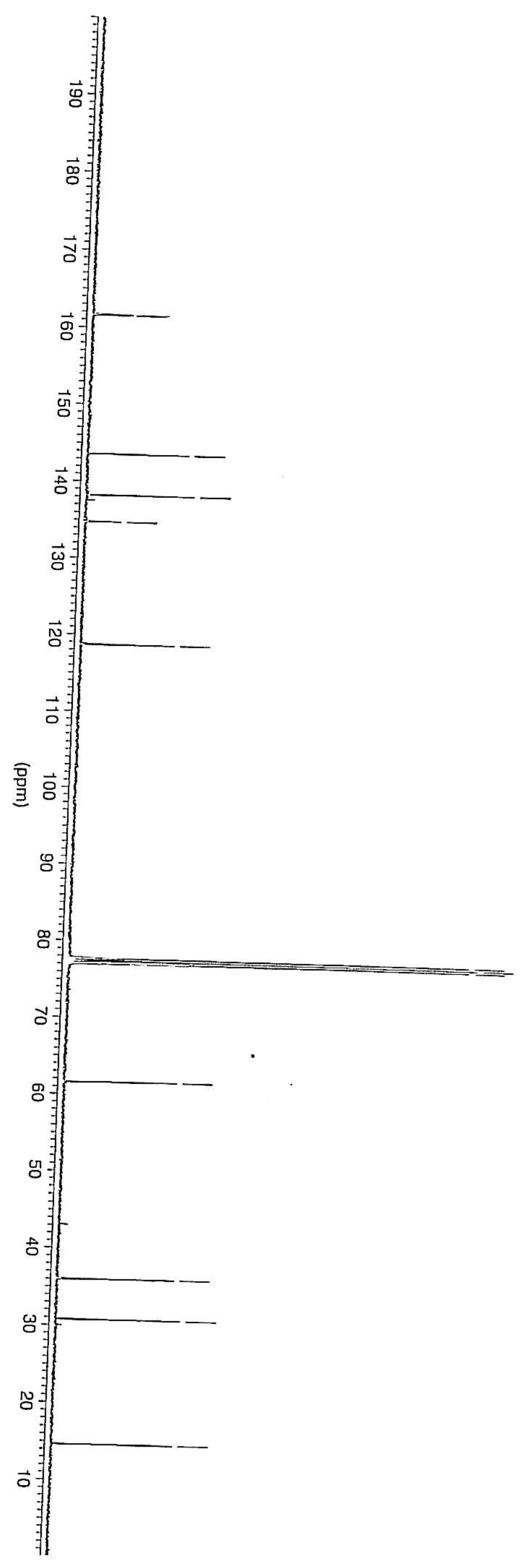

$\vec{n}$

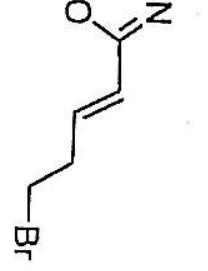

161.5663

161.4154

$-143.5578$

$-138.1902$

$-134.6806$

$-118.6413$

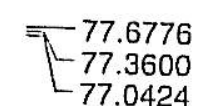

$-61.6145$

$-35.9834$

$-30.7825$

$-14.6321$ 

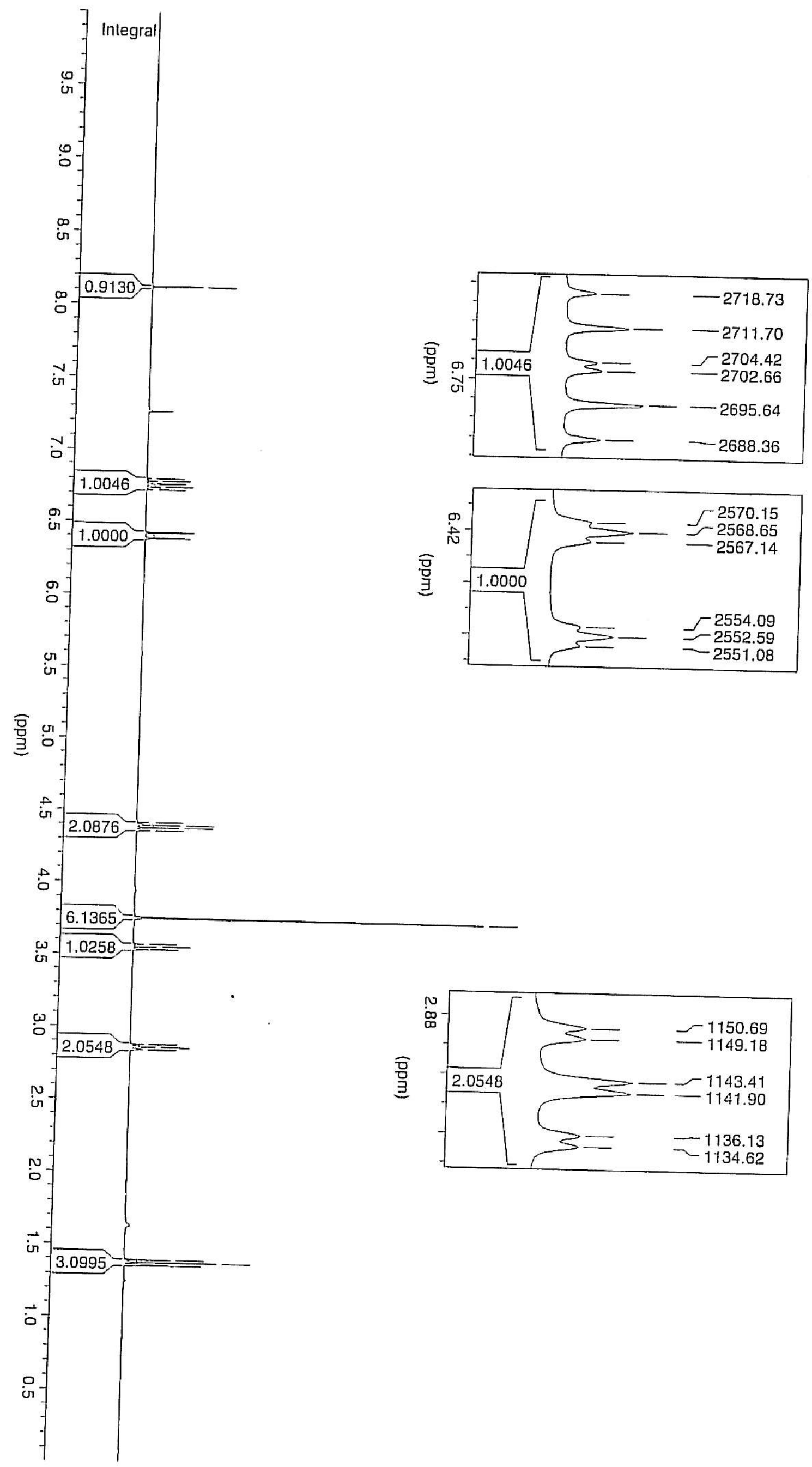

$-8.1130$
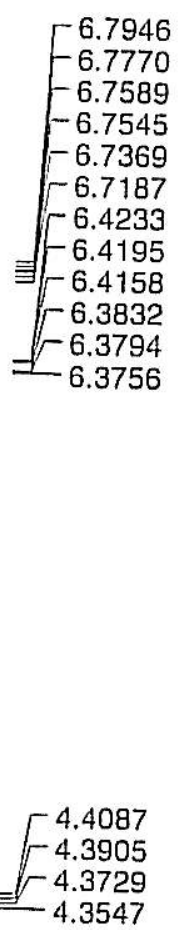

$-3.7482$

־ 3.5651

$-3.5469$

3.5281
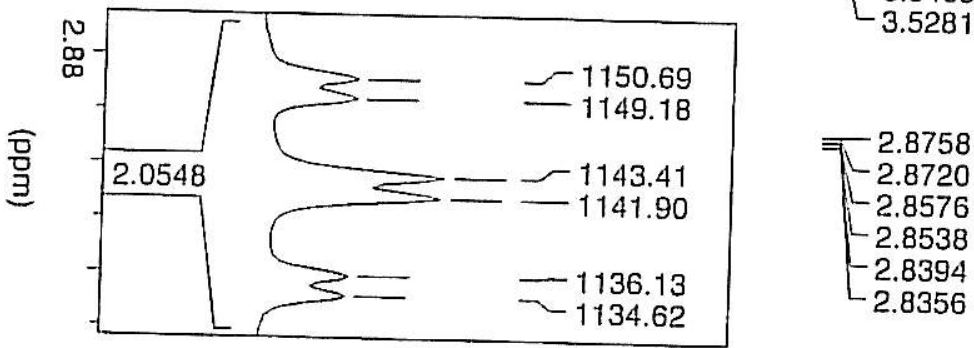

-1.3905

$-1.3730$

1.3548 

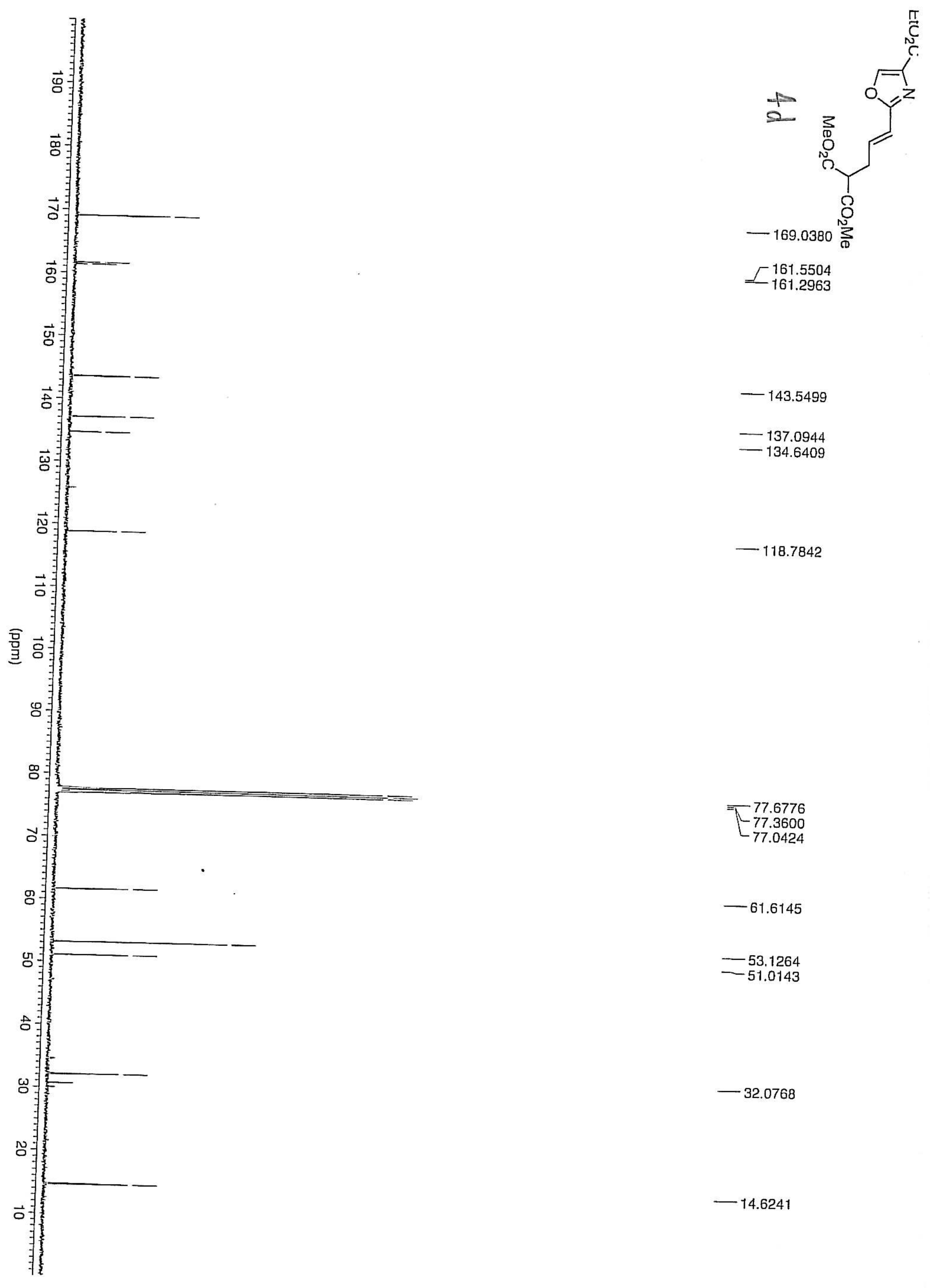

$-137.0944$

$-134.6409$

$-118.7842$

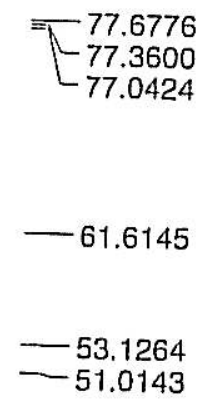

$-32.0768$

$-14.6241$ 

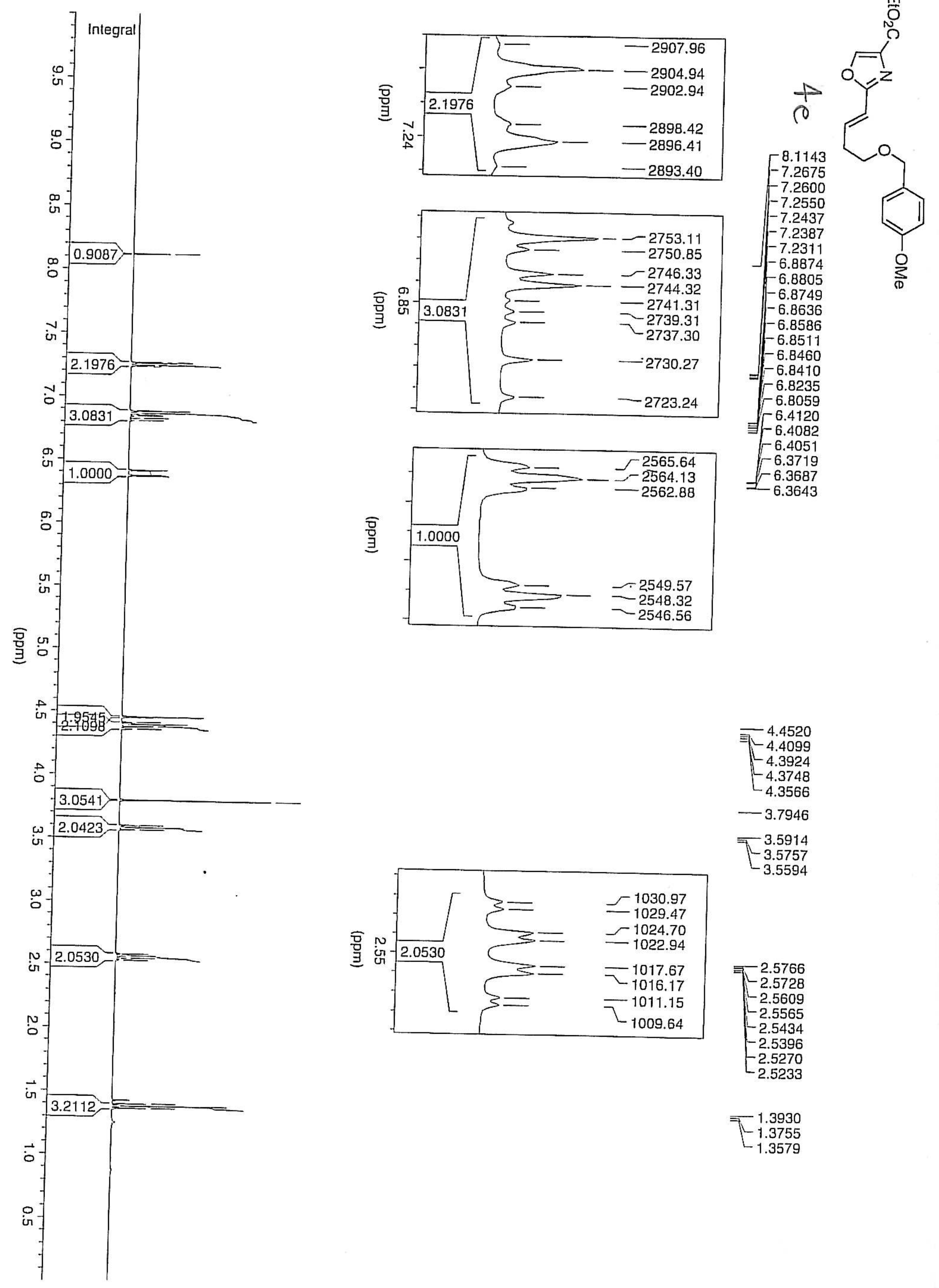

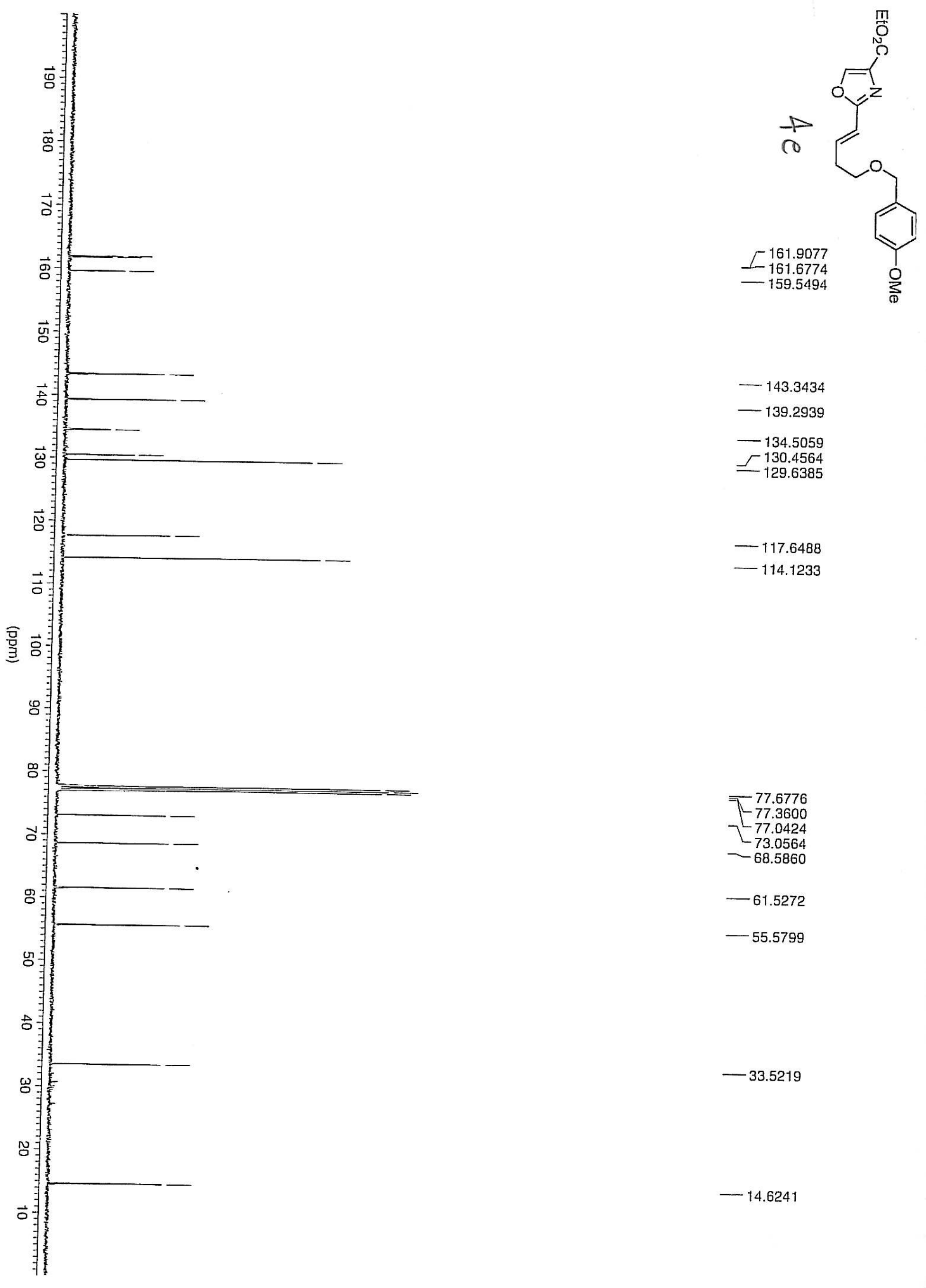

$-143.3434$

$-139.2939$

$-134.5059$

$\Gamma 130.4564$

129.6385

$-117.6488$

$-114.1233$

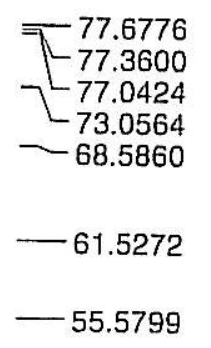

$-33.5219$

$-14.6241$ 

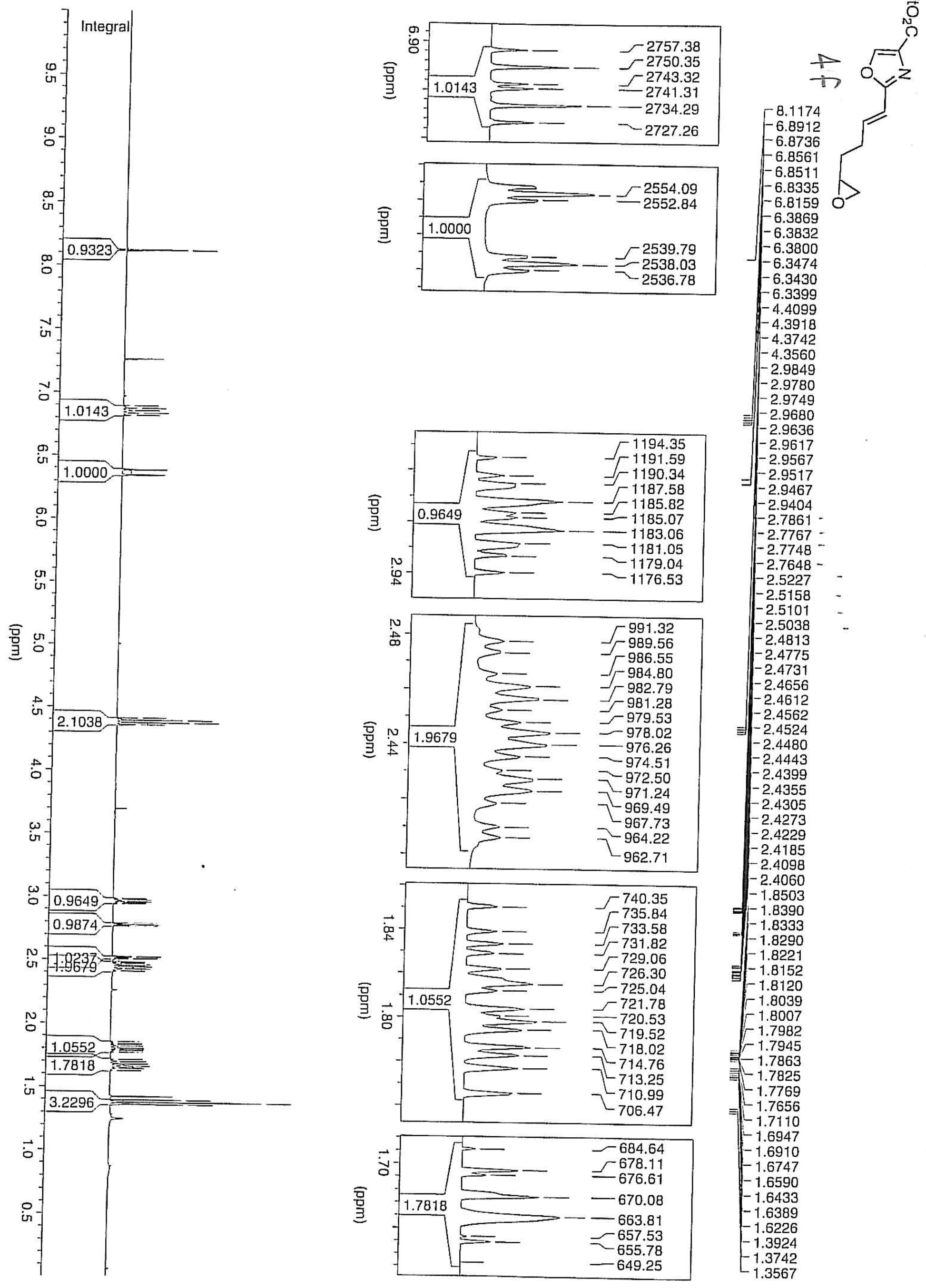


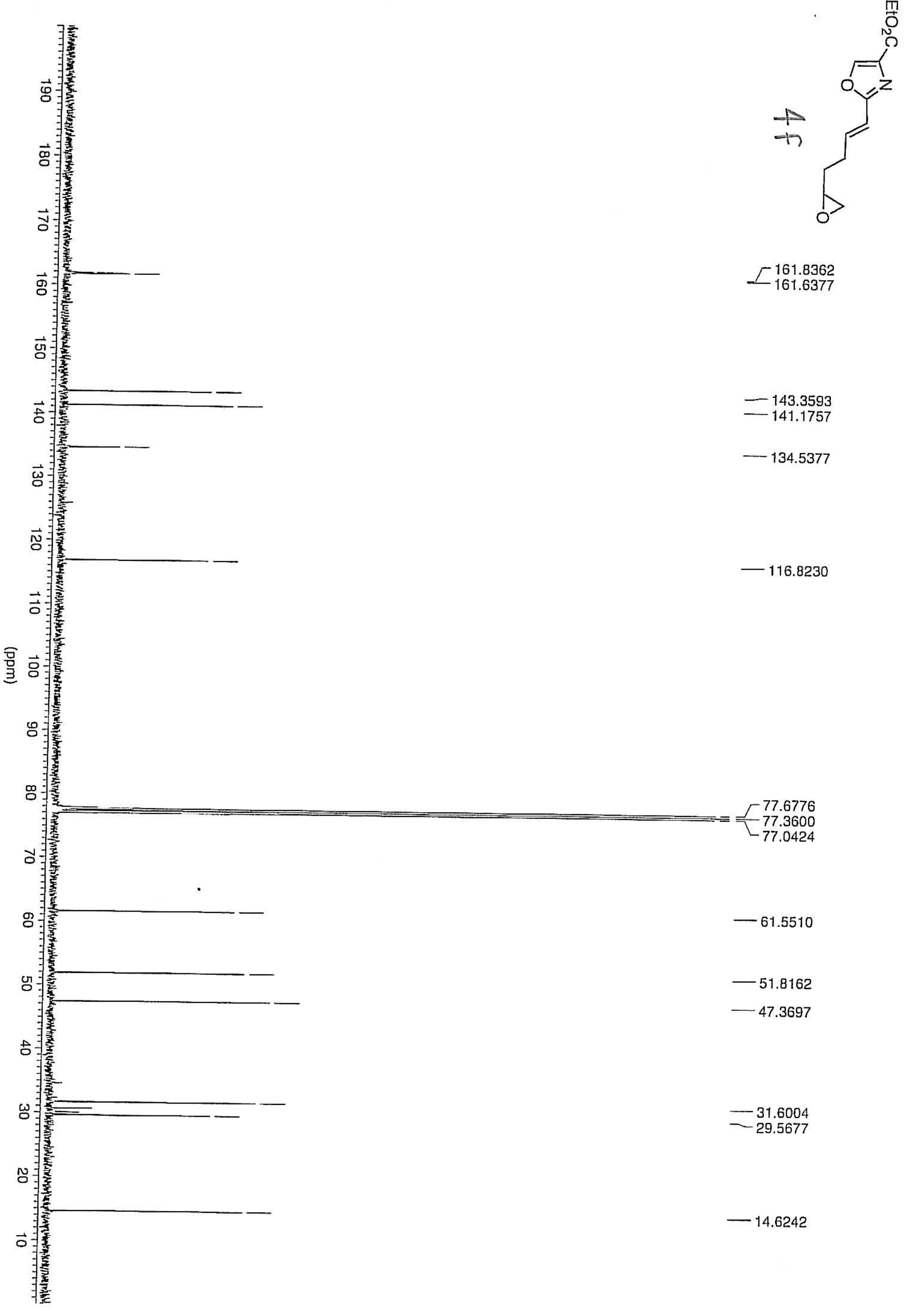



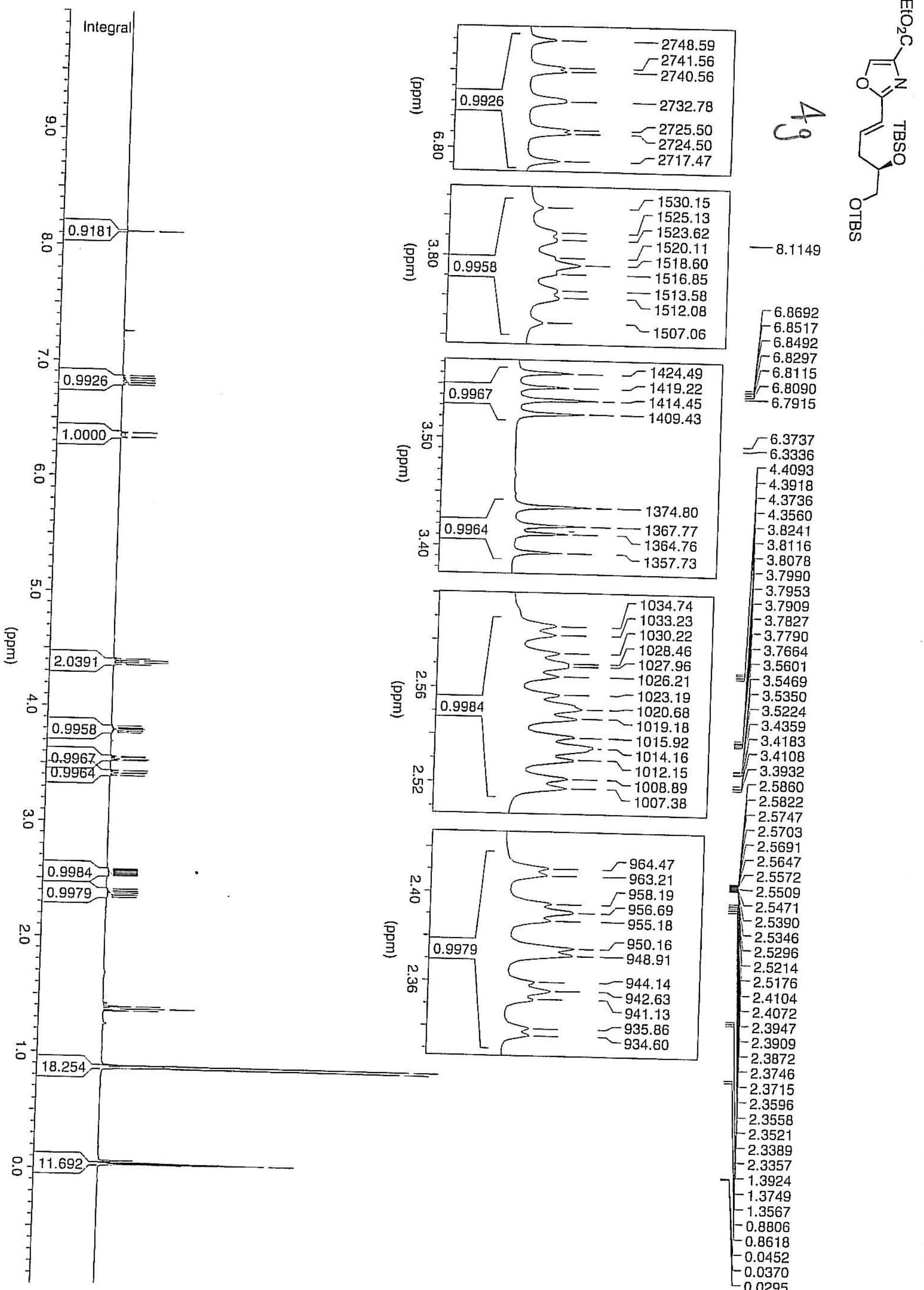

-6.8517
-6.8492

$-6.8297$

$-6.8115$

$-6.8090$

6.7915

$\Gamma^{6.3737}$

6.3336

4.4093

$-4.3918$

$-4.3736$

$-4.3560$

$-3.8241$

$-3.8116$

$-3.8078$

$-3.7990$

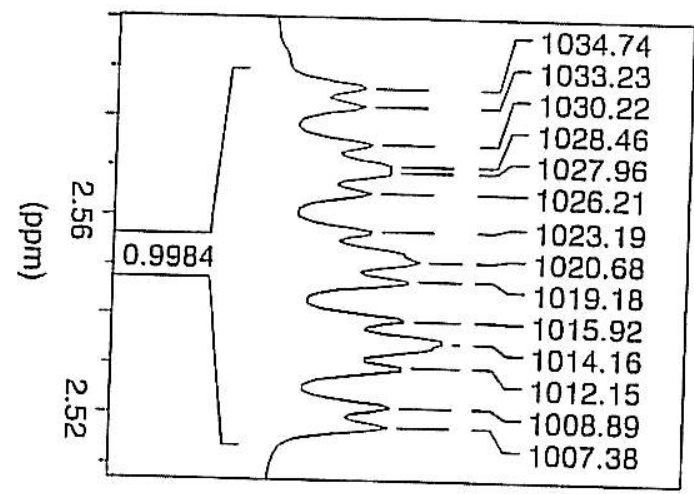

$-3.7953$

$-3.7909$

$-3.7827$

$-3.7790$

$-3.7664$

$-3.5601$

- 3.5469

- 3.5350

- 3.5224

$-3.4359$

$-3.4183$

$-3.4108$

$=-3.3932$

$=2.5860$

$-2.5822$

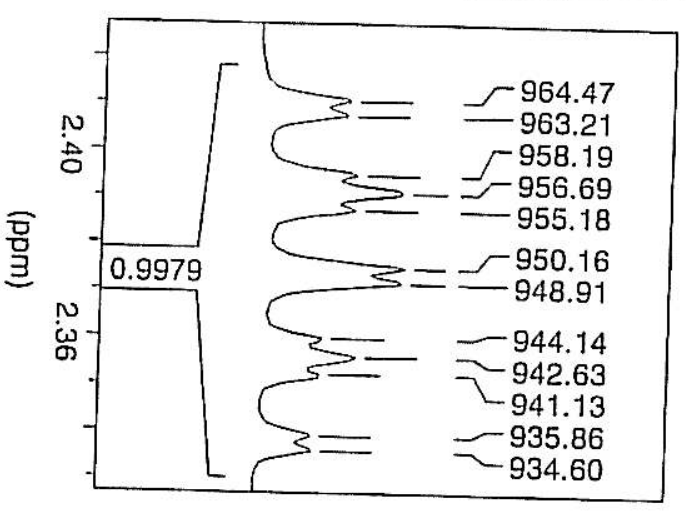

$-2.5747$

$-2.5703$

$-2.569$

$-2.5647$

$-2.5572$

$5-2.5509$

或 2.5471

L 2.5390

$-2.5346$

- 2.5296

$-2.5214$

$-2.5176$

$-2.4104$

$-2.4072$

$=-2.3947$

$-2.3872$

$-2.3746$

$-2.3715$

$-2.3596$

$-2.3558$

$-2.3521$

$-2.3389$

$-2.3357$

ᄀ- -1.3924

$-1.3749$

$-1.3567$

$-0.8806$

$-0.8618$

$-0.0452$

$-0.0370$

$-0.0295$ 

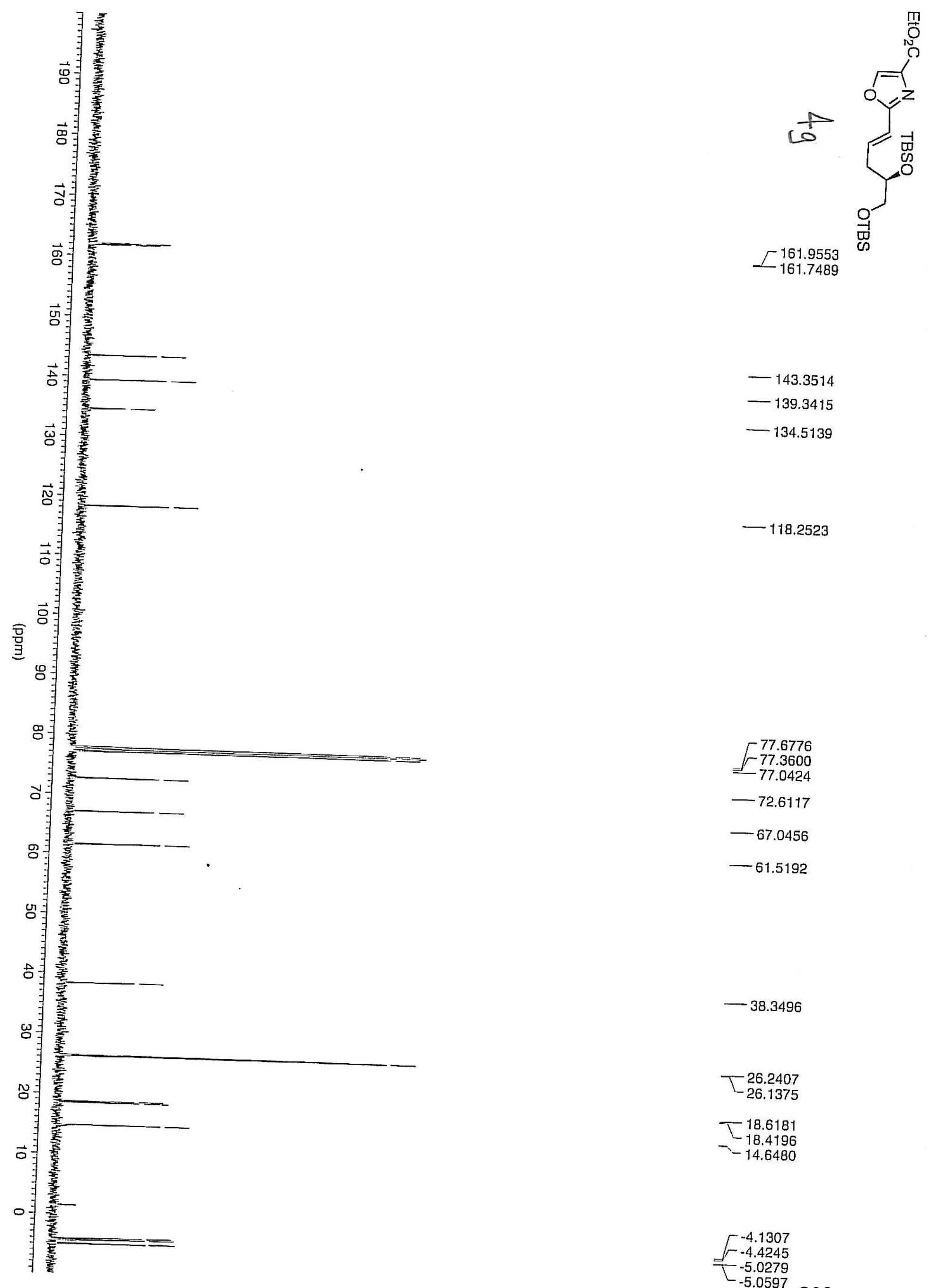

$-143.3514$

$-139.3415$

$-134.5139$

$-118.2523$

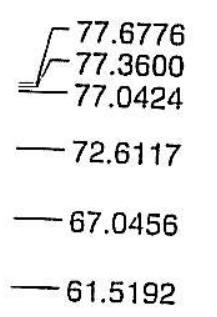

$-38.3496$

$-26.2407$

26.1375

18.6181
-18.4196
-14.6480

$-14.6480$

$F^{-4.1307}$

$-\begin{array}{r}-5.0279 \\ -5.0597\end{array}$ 


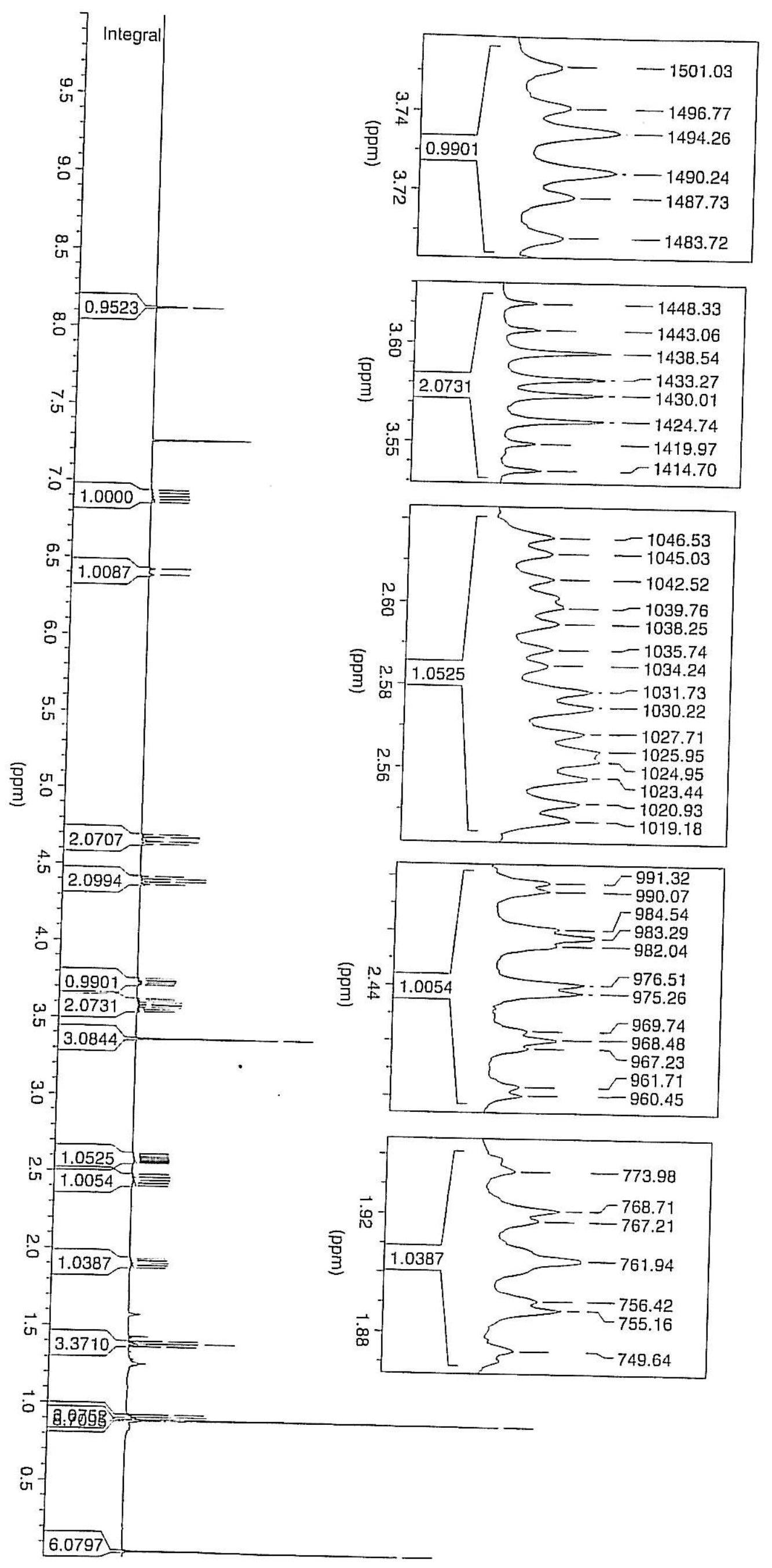




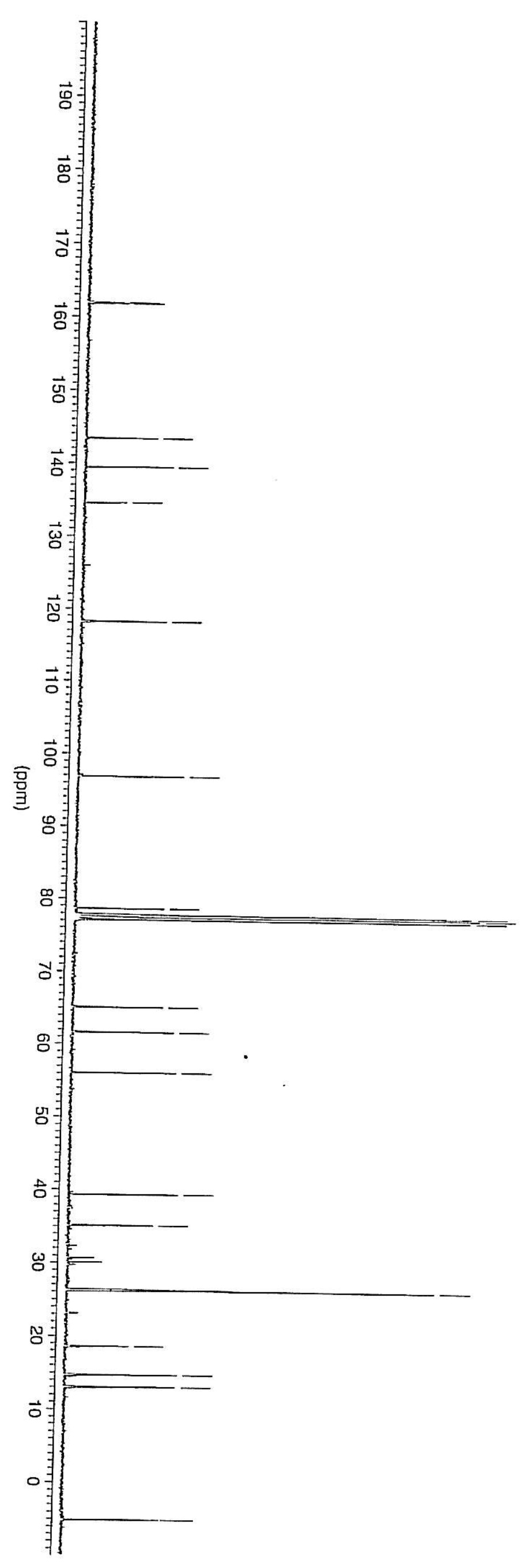



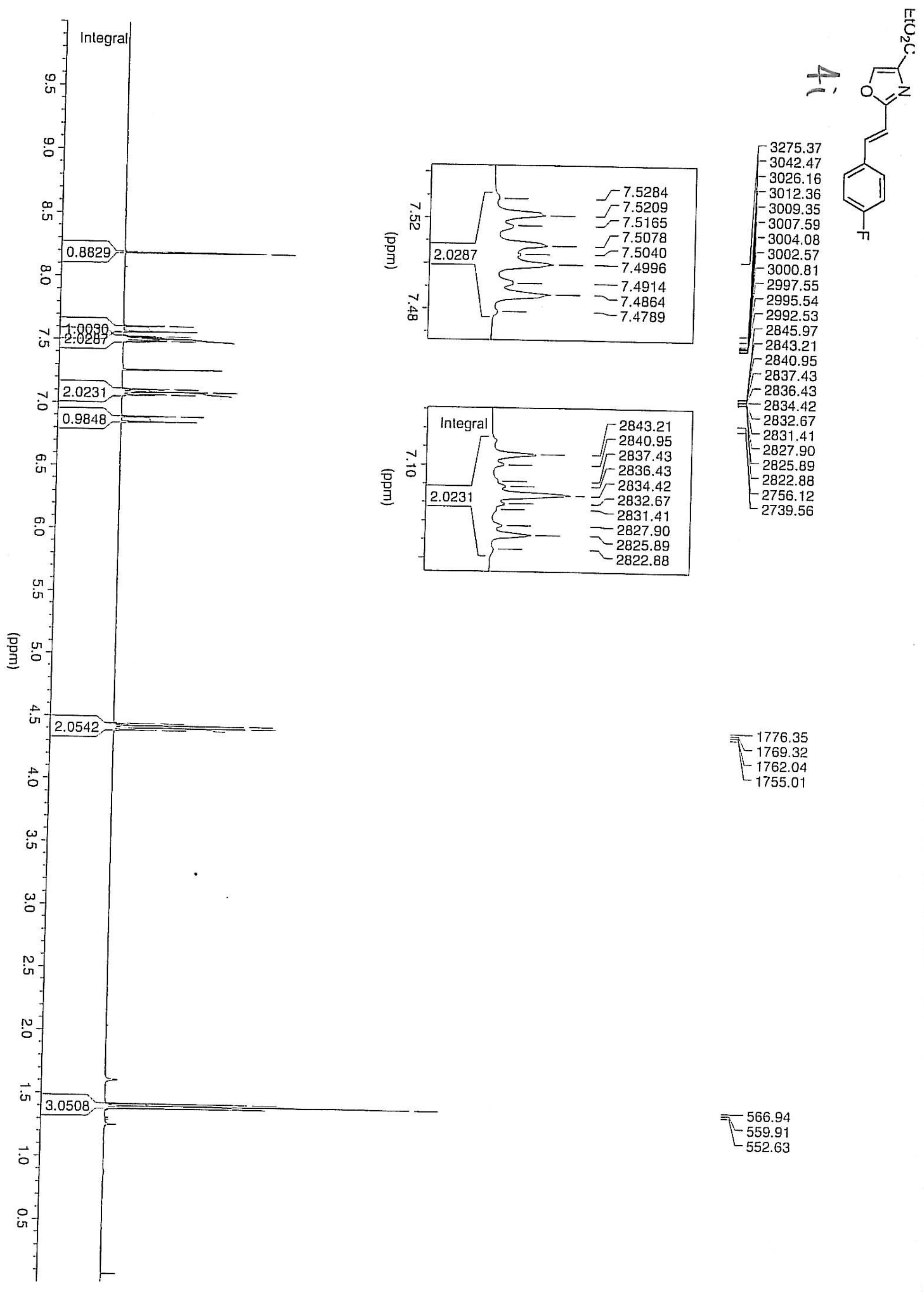

- 1776.35

$-1769.32$

$-1762.04$

$-1755.01$ 


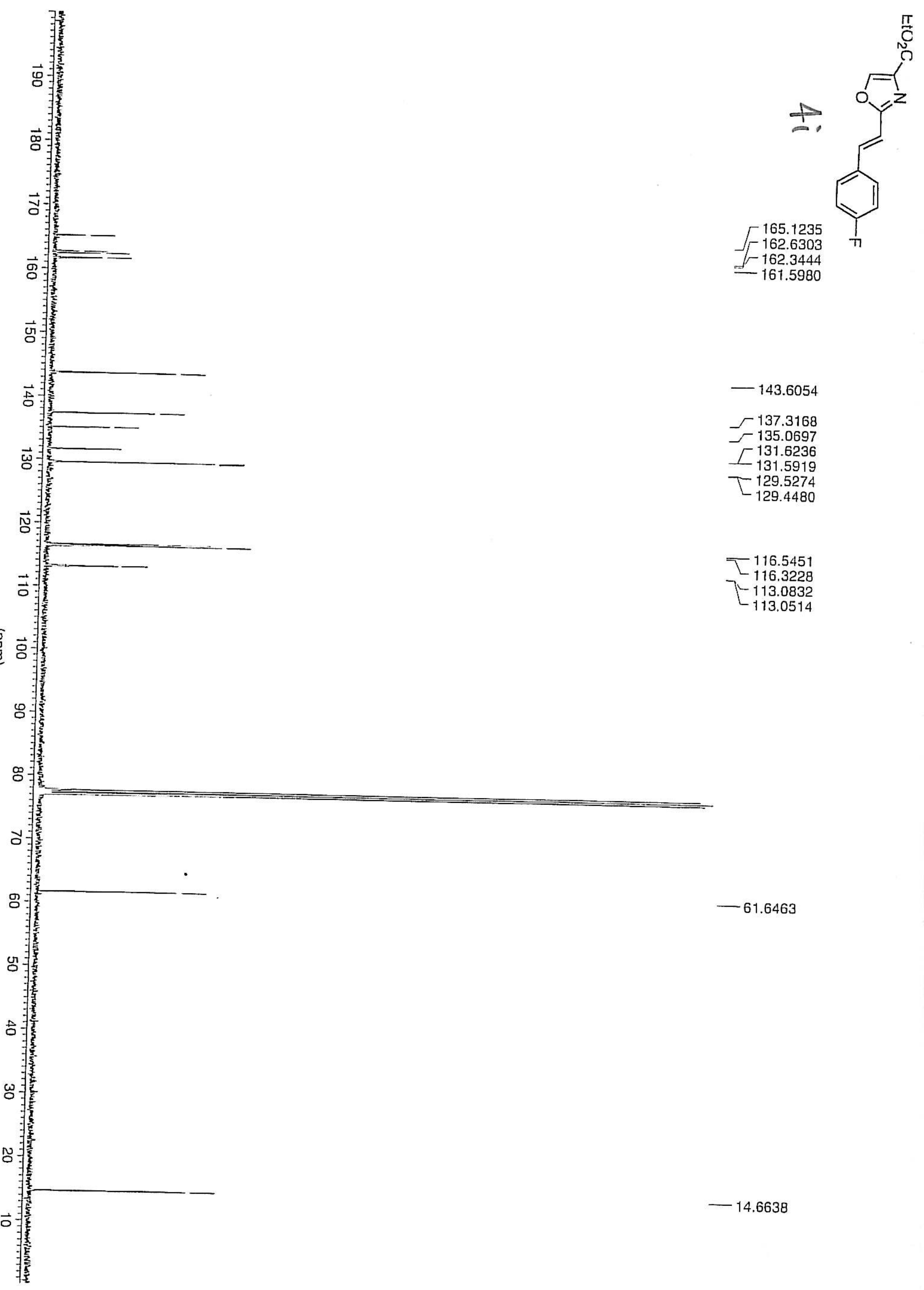




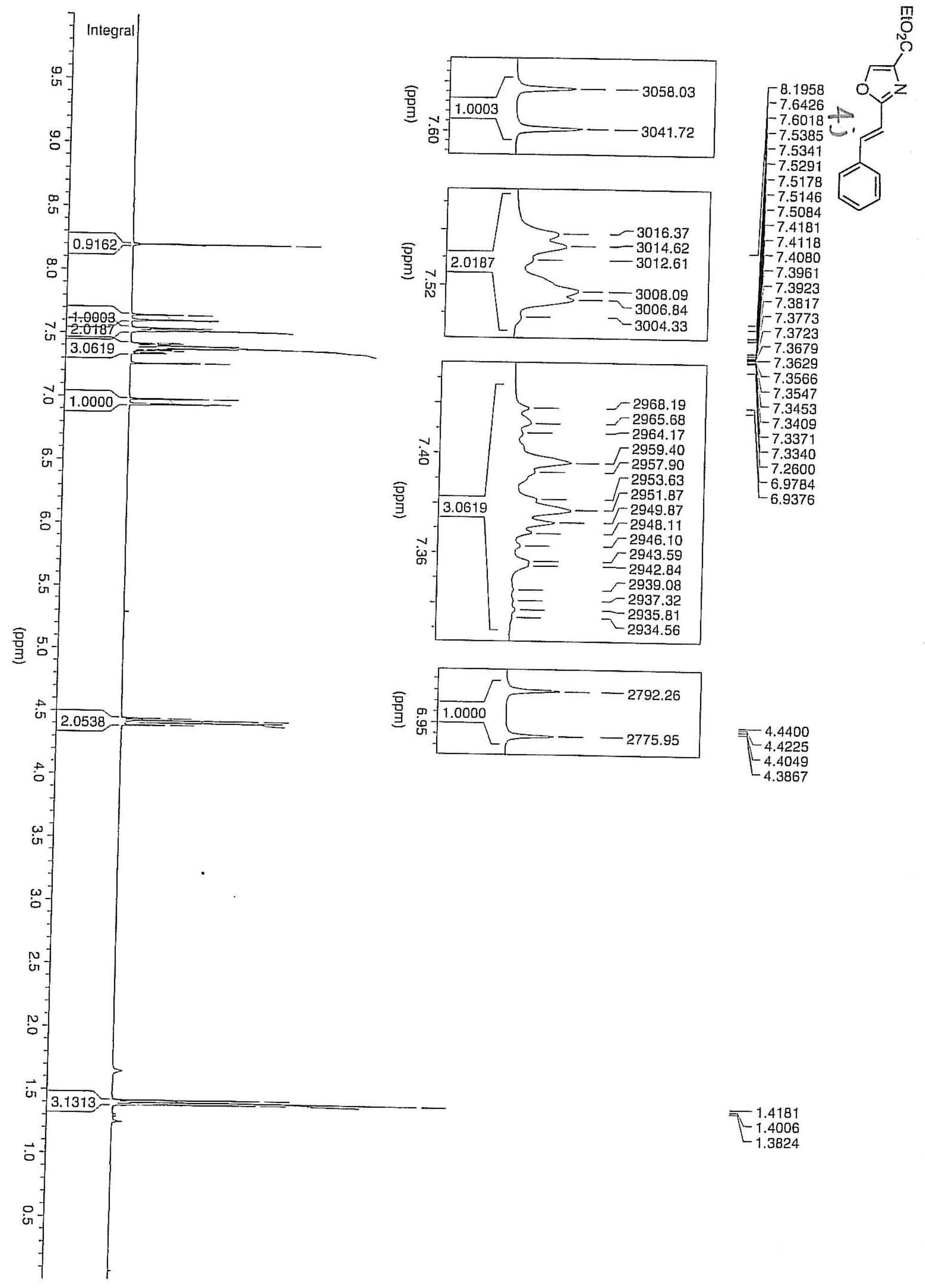




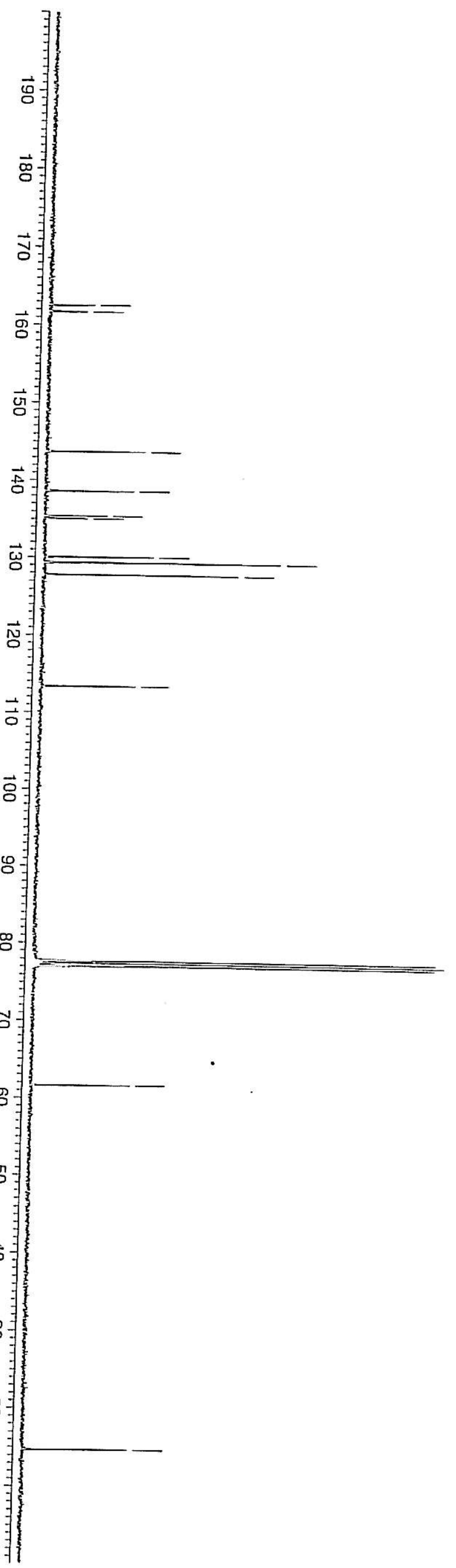




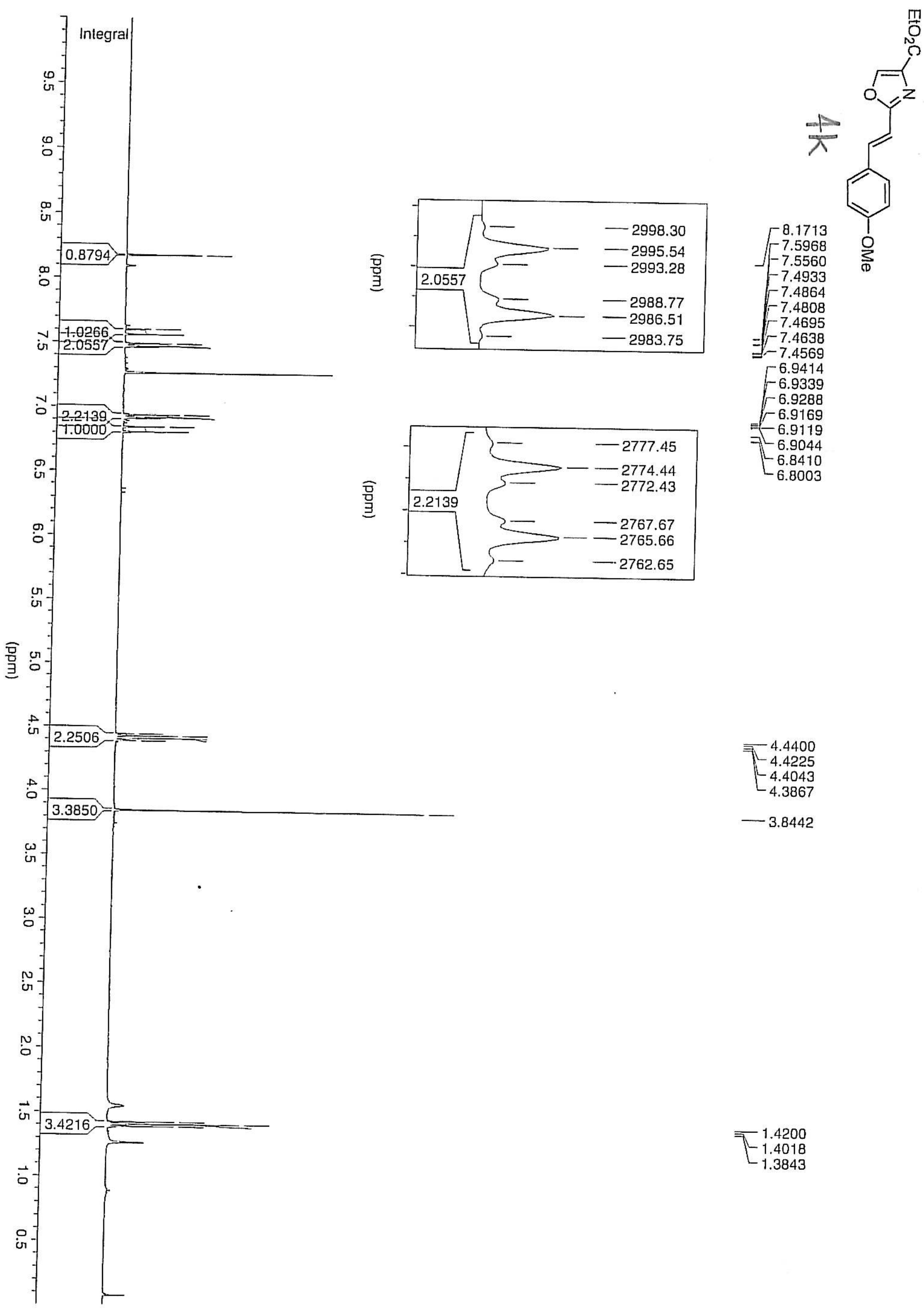




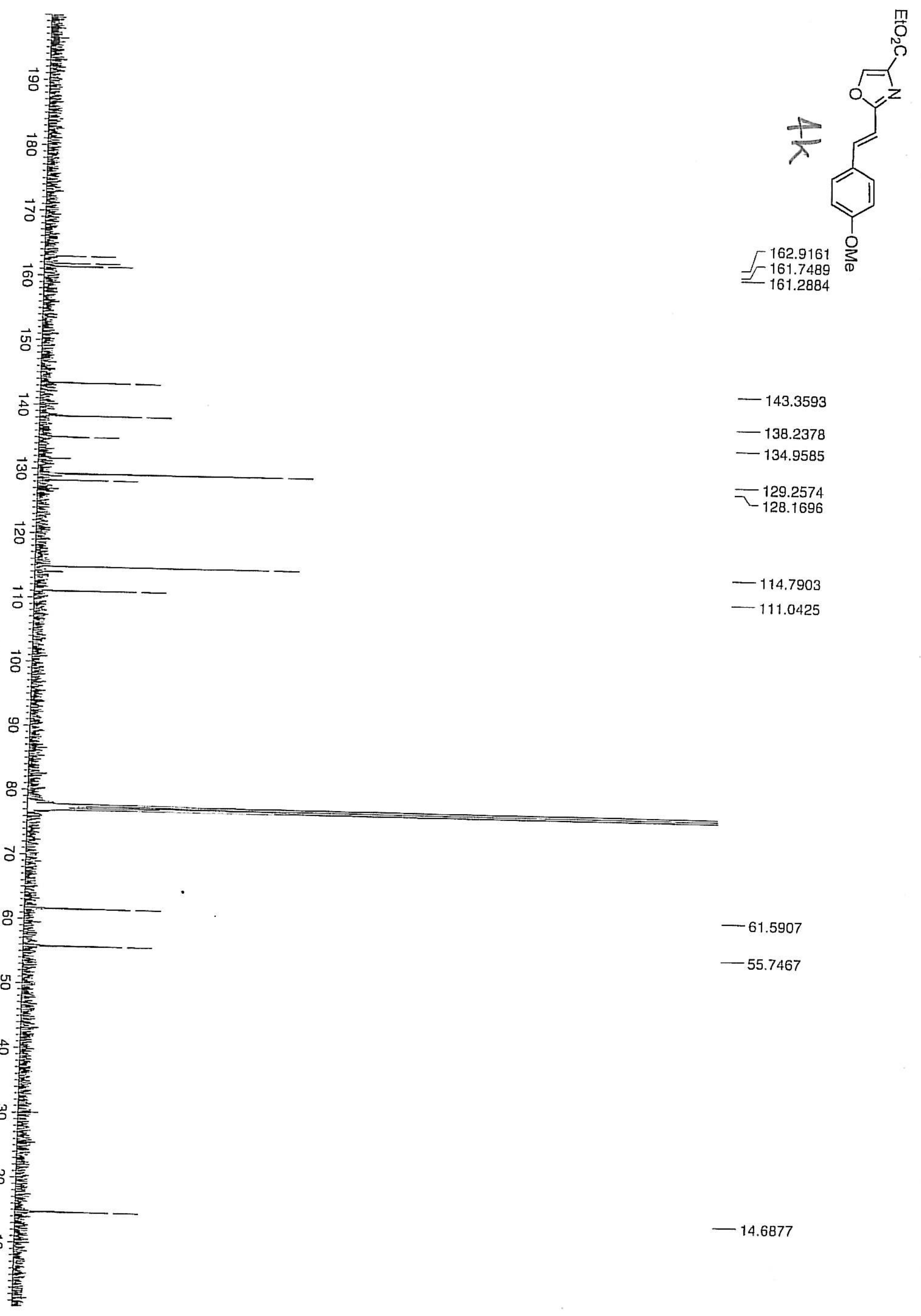




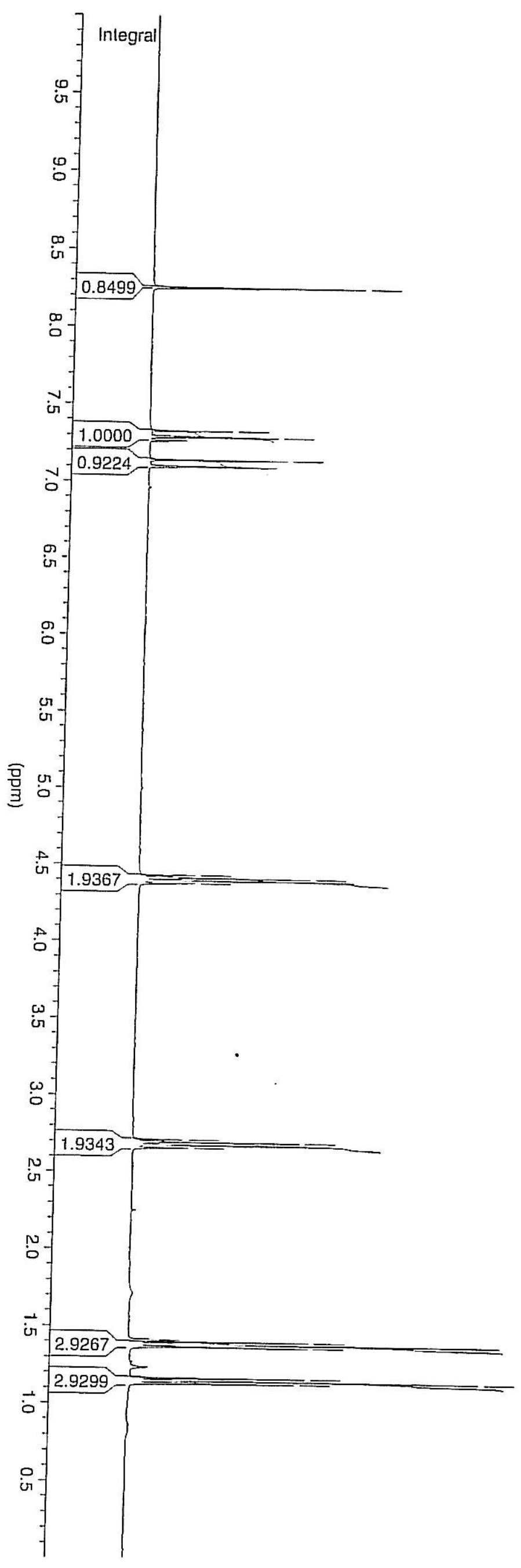




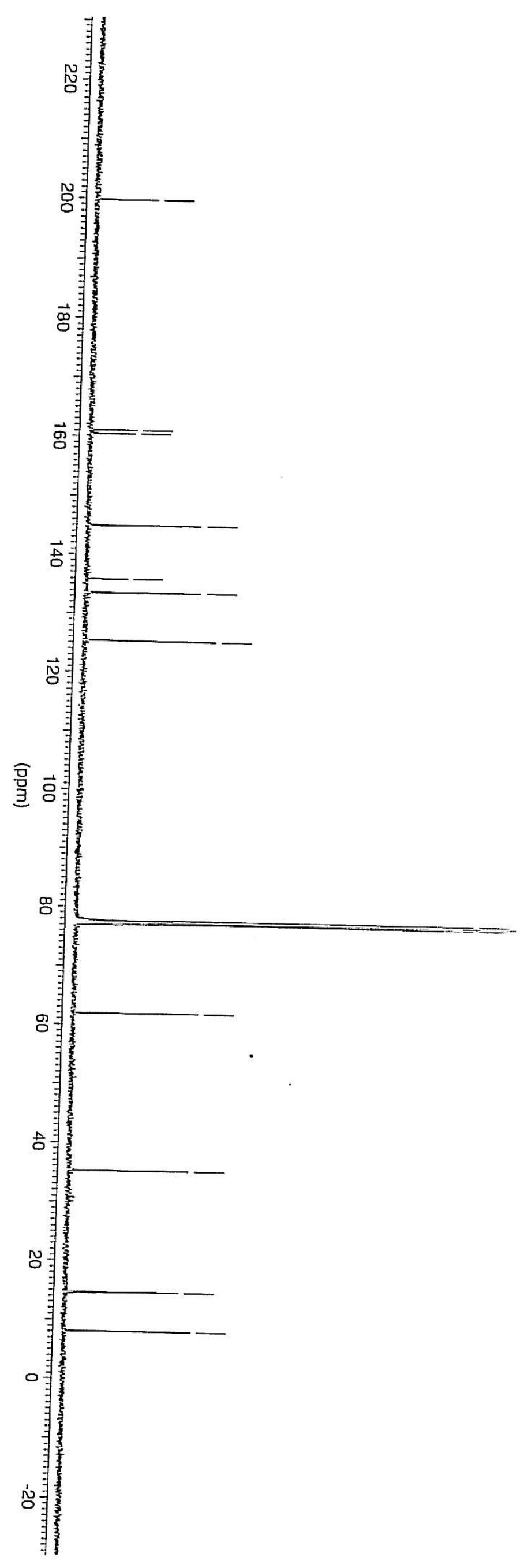

161.0343
-160.4149

$-144.9553$

-135.8558
-133.4816

$-125.2714$ 


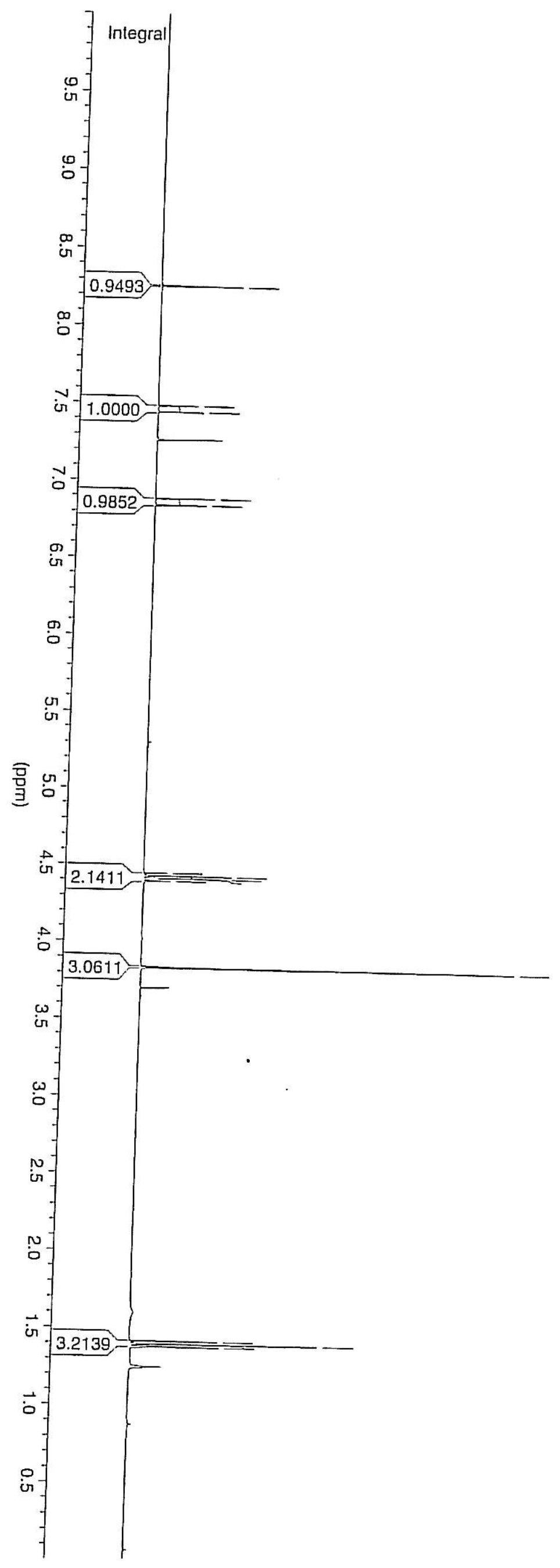



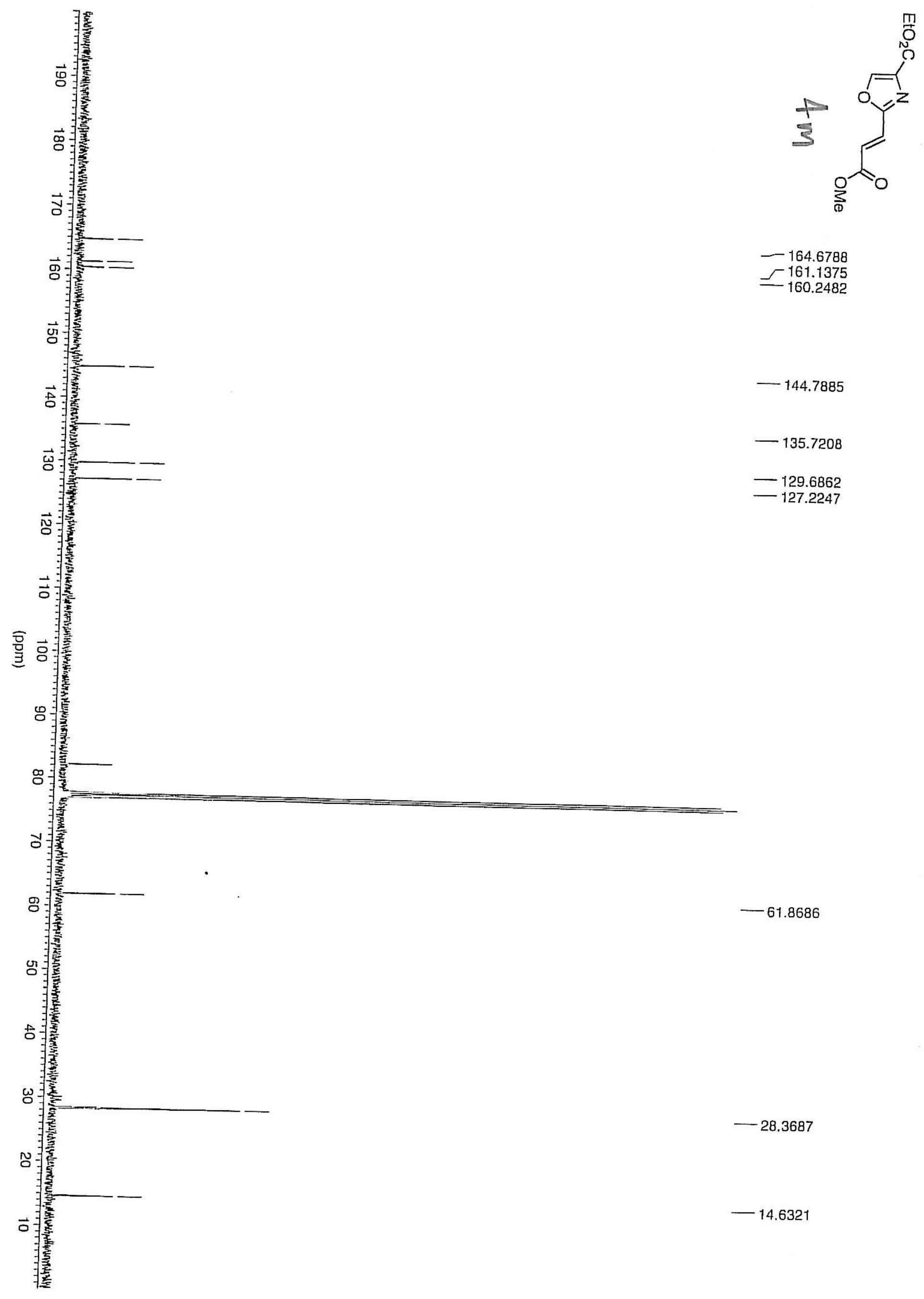

$-144.7885$

$-135.7208$

$-129.6862$

$-127.2247$ 


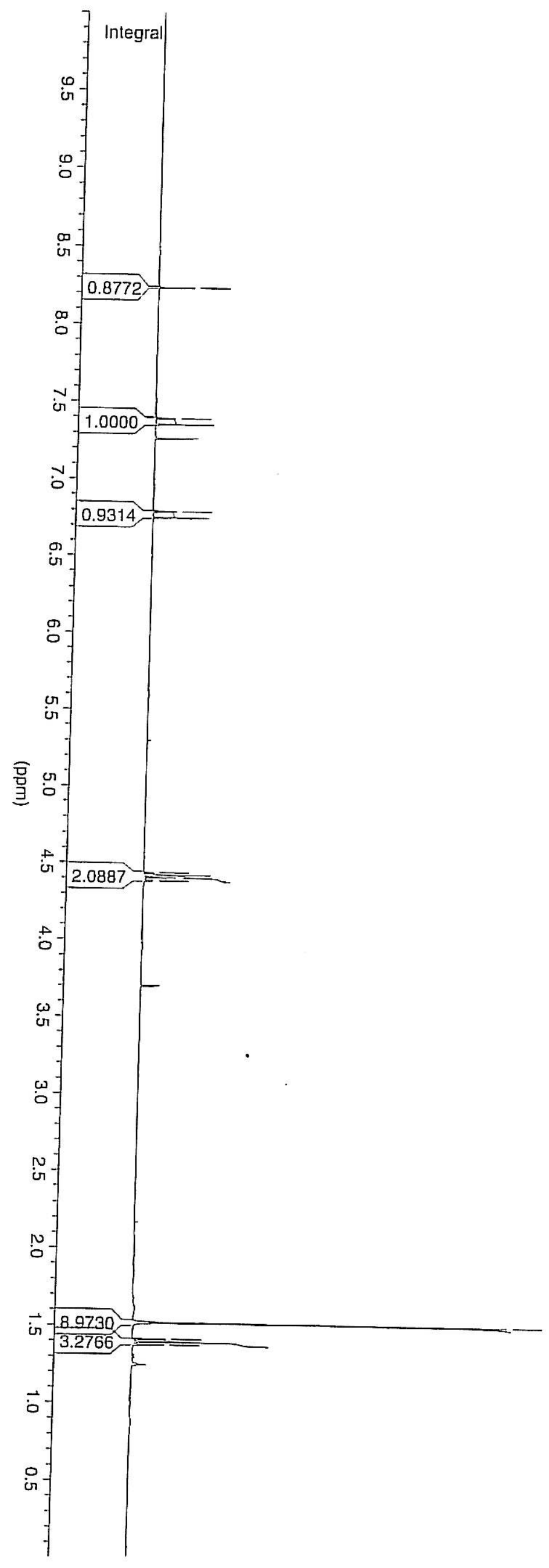

$-1.3755$ 
触<smiles>CC(C)(C)OC(=O)/C=C/c1[Z]c(O[Hg])co1</smiles>

$-165.9731$

$-161.0581$

형=ㅡㄹ

$-159.9465$

范䎏

$\vec{\Delta}$

$-144.9473$

$-135.8399$

$-128.3284$

127.2088

N.

$\overrightarrow{0}$

믈 훙

$8:$

果㐘

డे

秘

ช。

8.

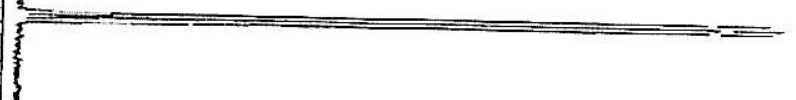

ॠ77.6776

$-77.0424$

$-61.9242$

$-52.6500$

당

接

埕

b.

$-30.0362$

N: 


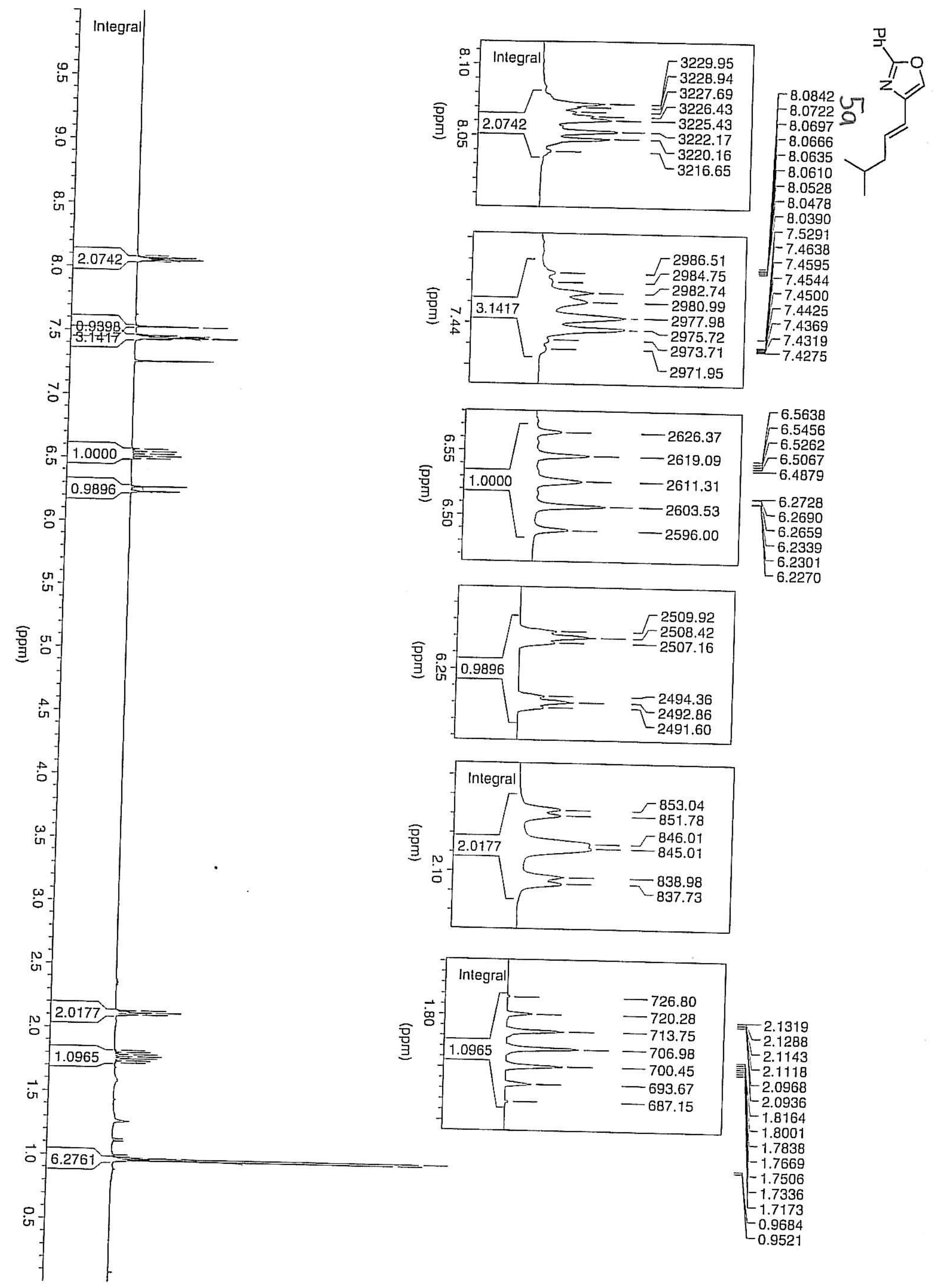




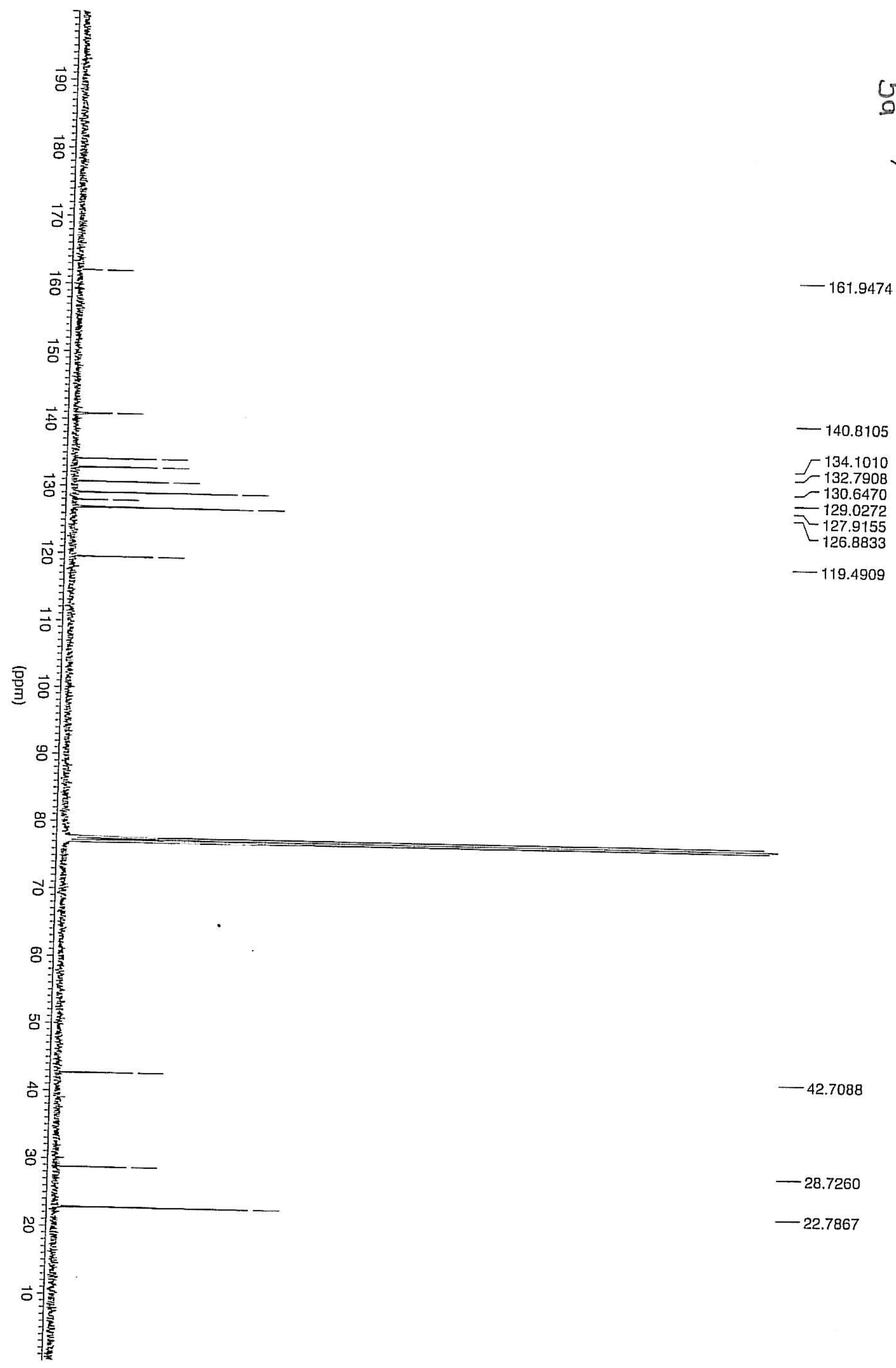




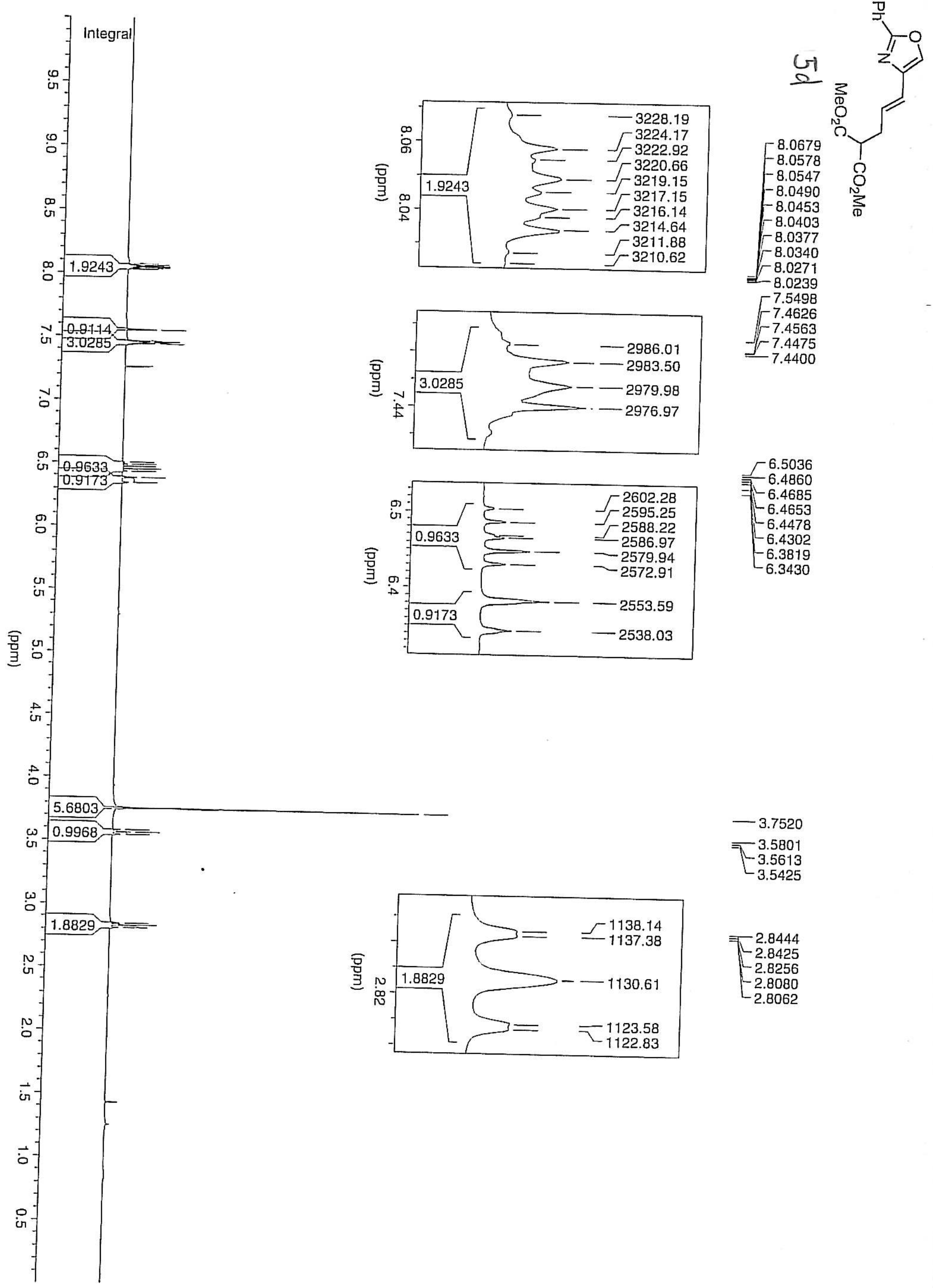




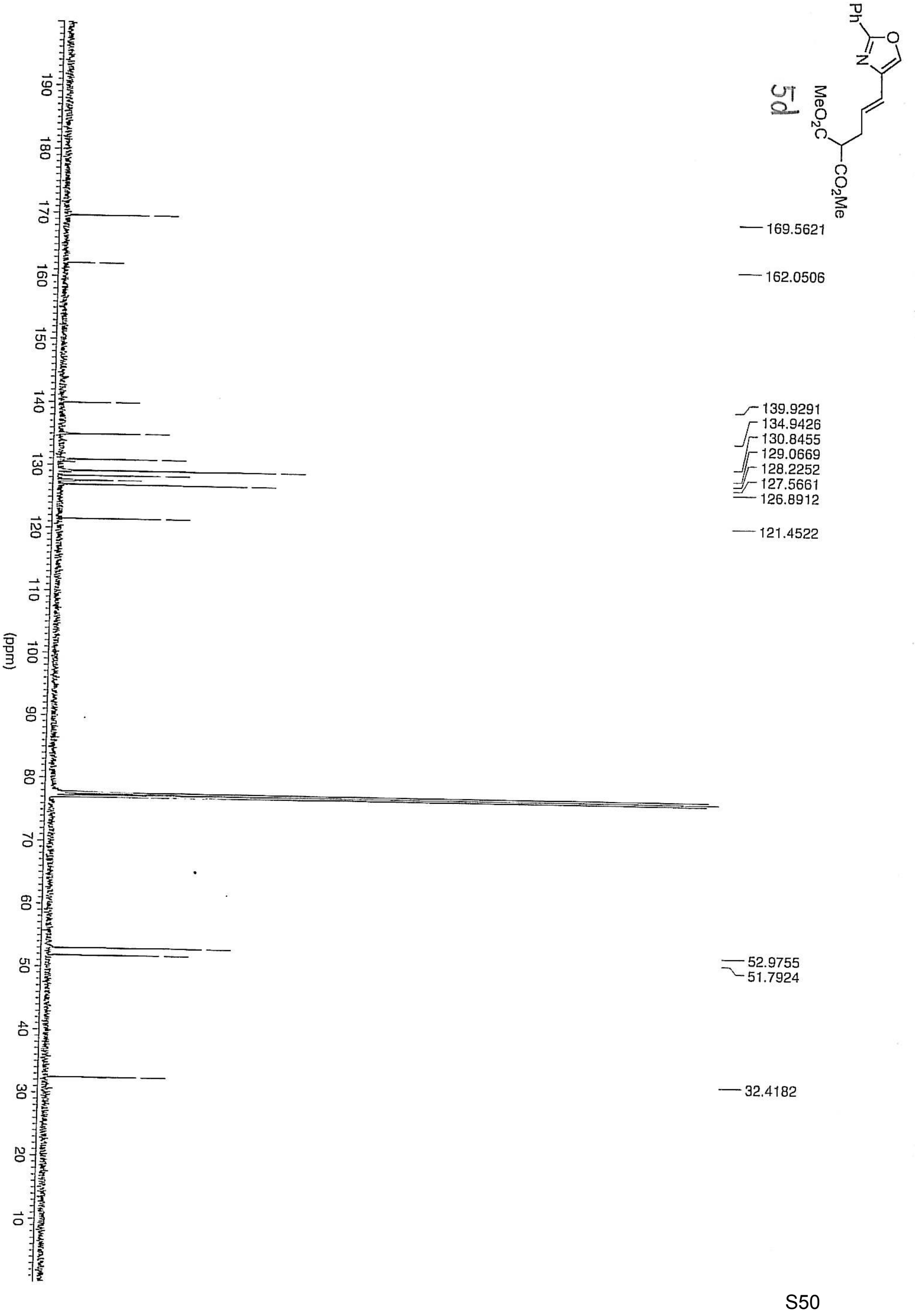




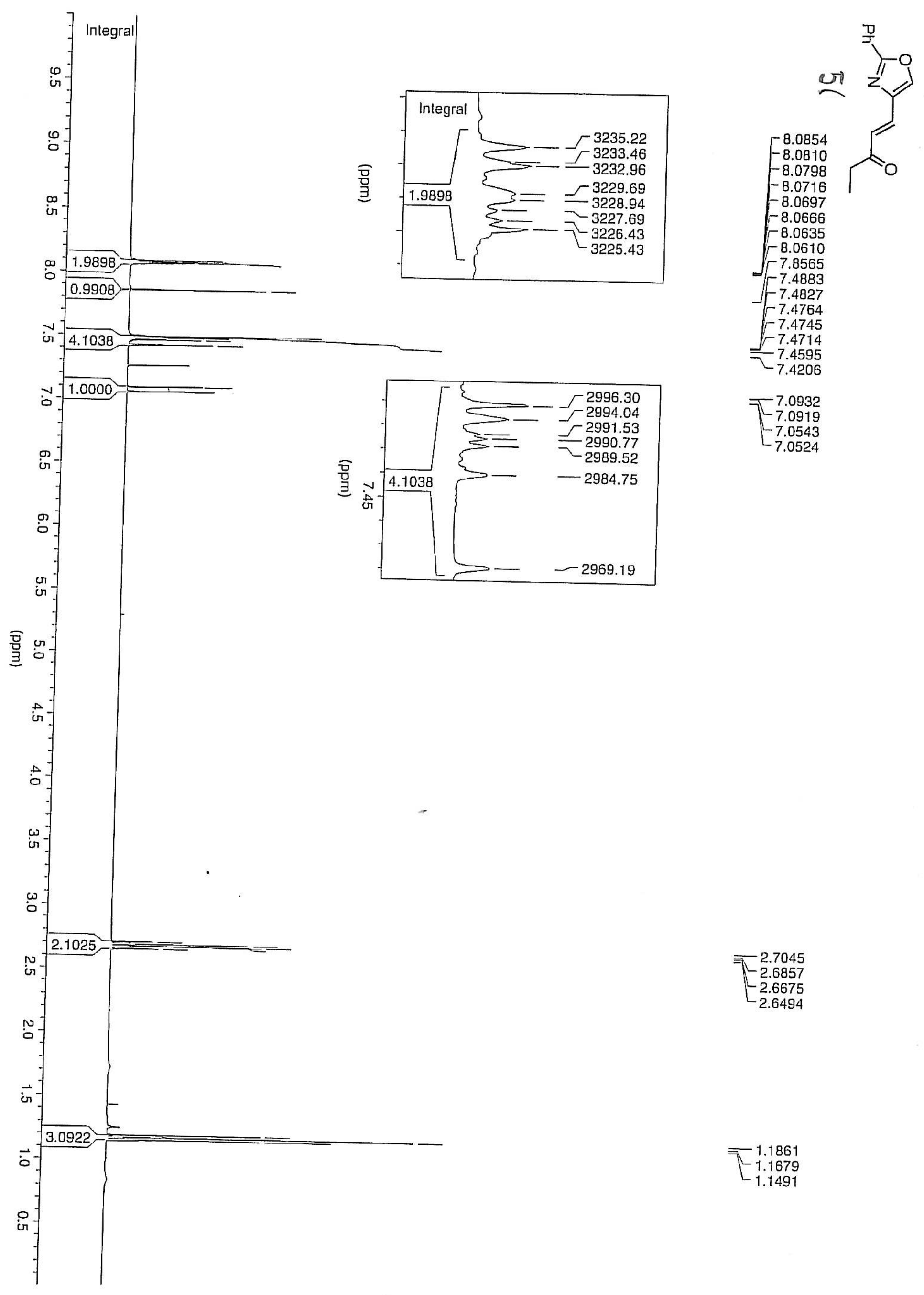




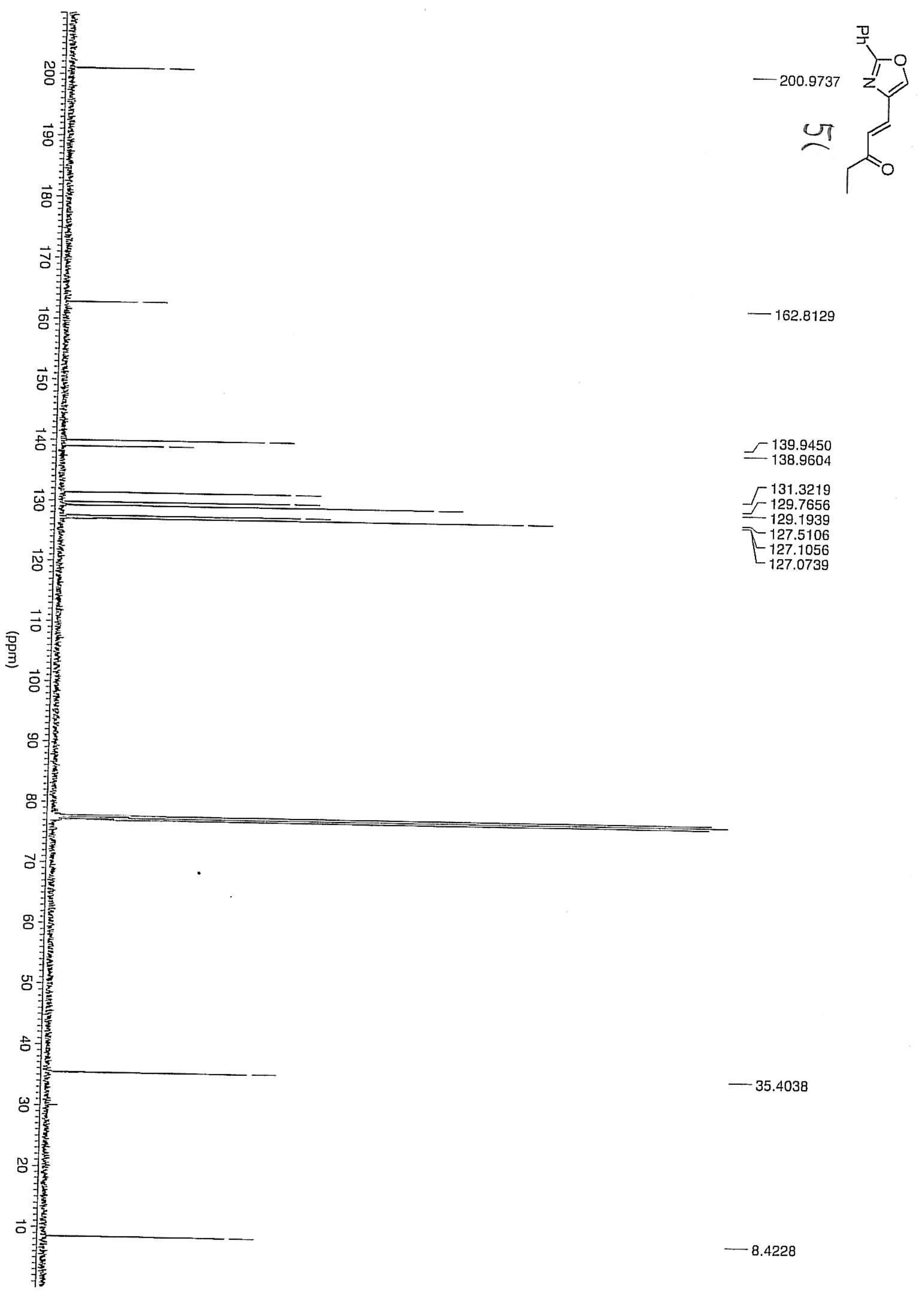




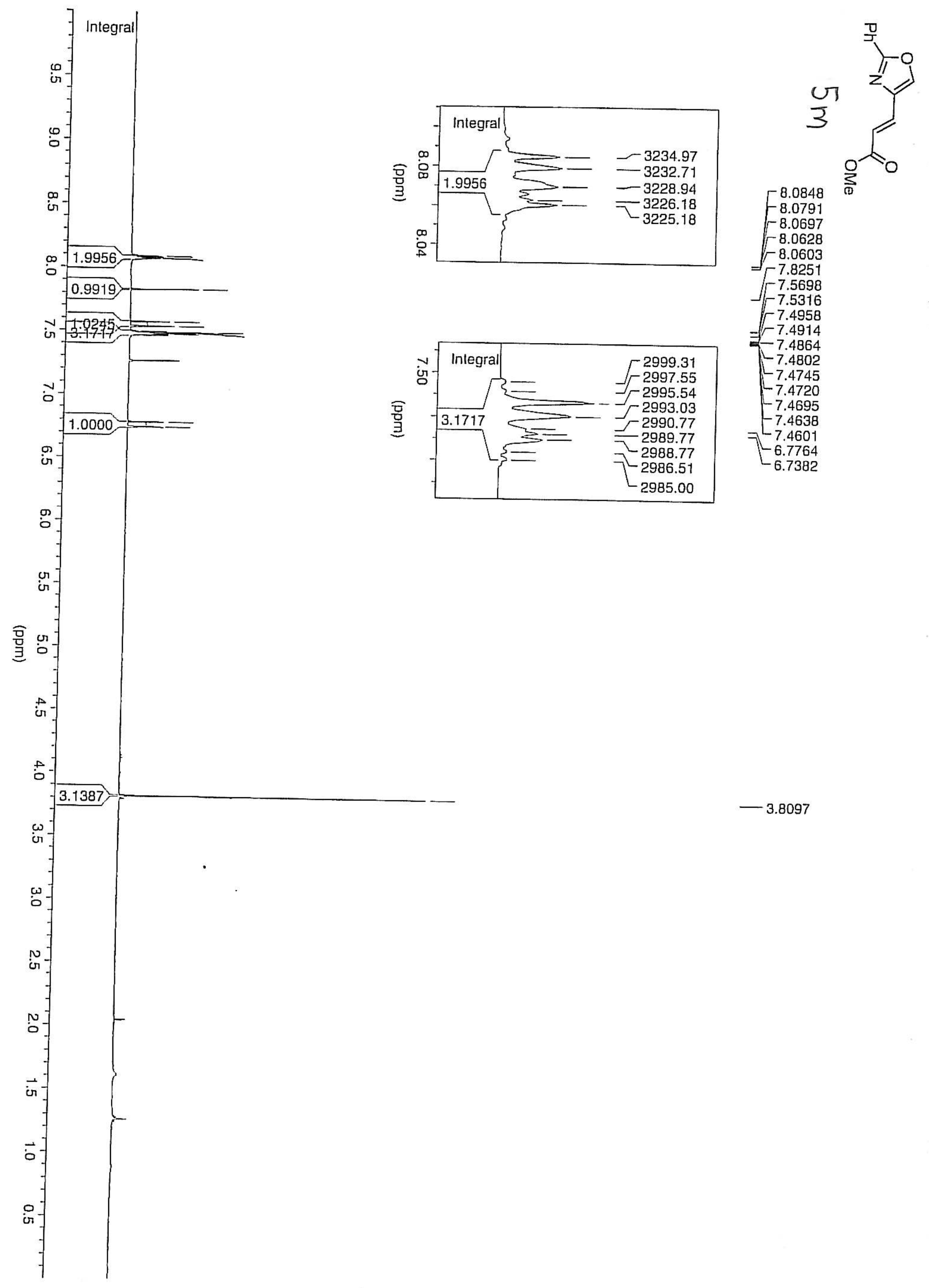




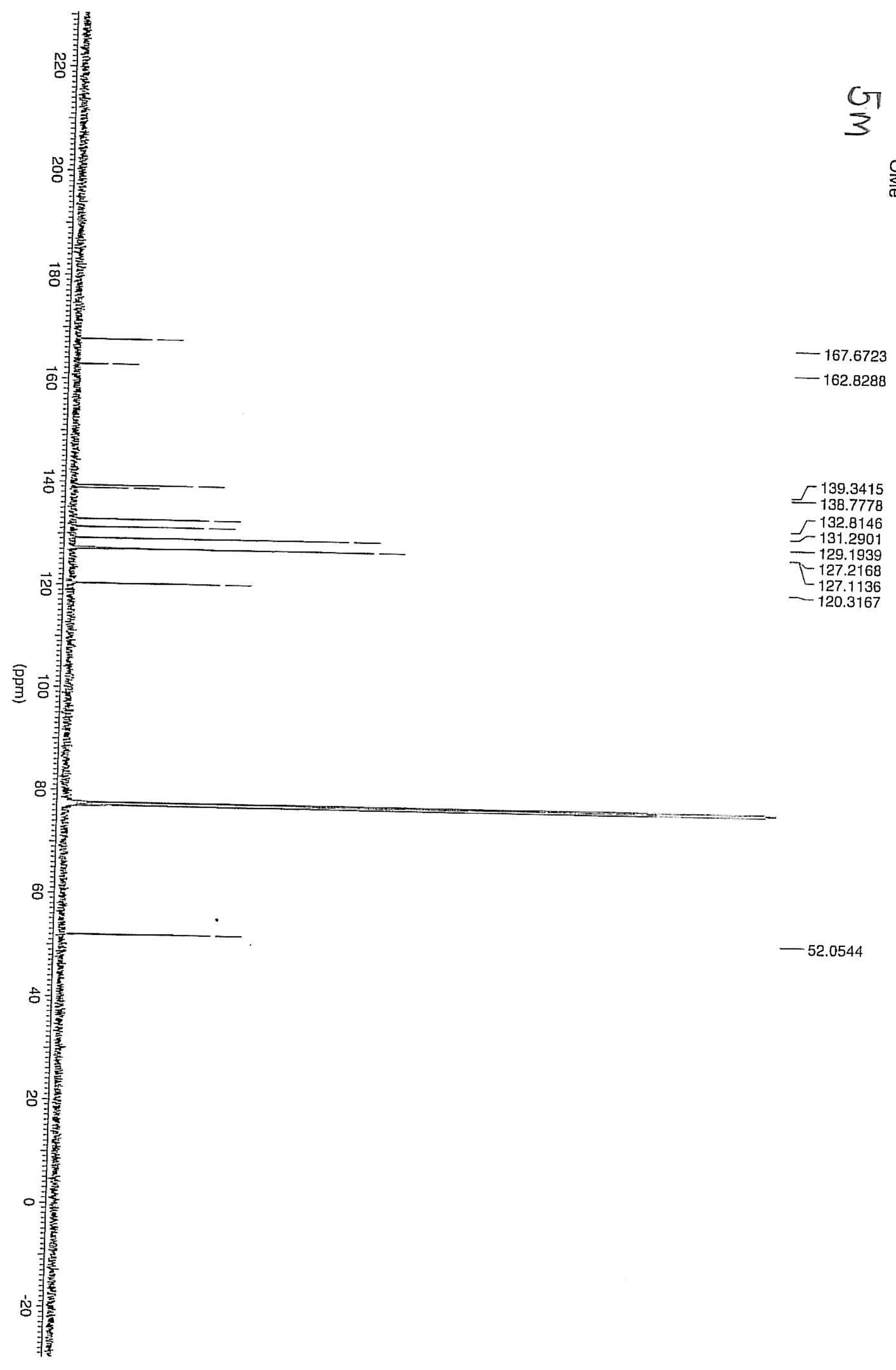




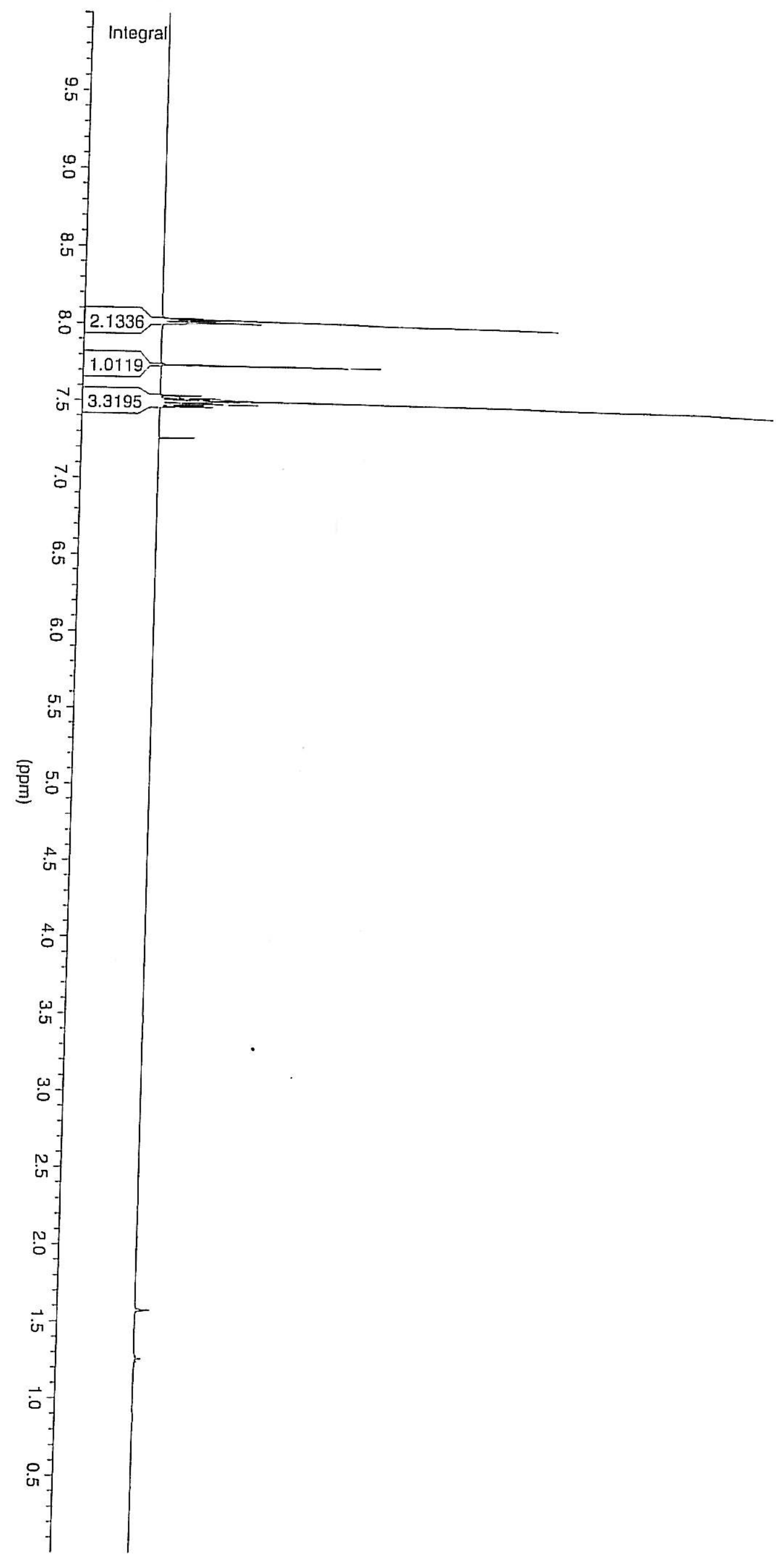

$\left[\begin{array}{r}8.0340 \\ -8.0277 \\ -8.0239 \\ -8.0189 \\ -8.0133 \\ -8.0083 \\ -8.0039 \\ -7.7354 \\ -7.5385 \\ -7.5353 \\ -7.5310 \\ -7.5284 \\ -7.5178 \\ 7.5153 \\ -7.5090 \\ -7.5046 \\ -7.4996 \\ -7.4983 \\ -7.4877 \\ -7.4833 \\ -7.4795 \\ -7.4726 \\ -7.4682 \\ -7.4638 \\ -7.4620 \\ 7.4576\end{array}\right.$<smiles>COc1coc([Hg])c1O</smiles> 


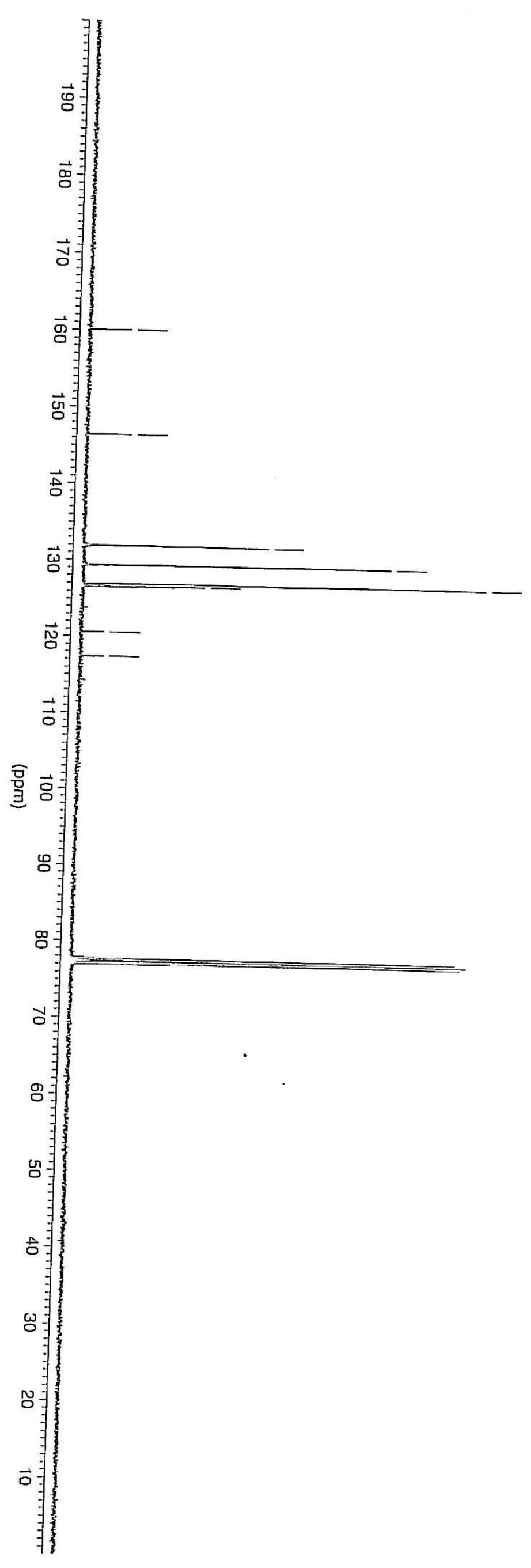

$-160.0259$

$-146.3369$

131.8618

$-129.2971$

- 126.9071

$-126.6213$

$-126.4625$

$-120.5708$

$-117.3788$ 